\title{
Supernovae and their host galaxies
}

\section{The SDSS DR8 database and statistics ${ }^{\star}$}

\author{
A. A. Hakobyan ${ }^{1,2,3}$, V. Zh. Adibekyan ${ }^{4}$, L. S. Aramyan ${ }^{1,2,3}$, A. R. Petrosian ${ }^{1,3}$, J. M. Gomes ${ }^{4}$, \\ G. A. Mamon ${ }^{5}$, D. Kunth ${ }^{5}$, and M. Turatto
}

\author{
1 Byurakan Astrophysical Observatory, 0213 Byurakan, Aragatsotn province, Armenia \\ e-mail: hakobyan@bao.sci.am \\ 2 Department of General Physics and Astrophysics, Yerevan State University, 1 Alex Manoogian, 0025 Yerevan, Armenia \\ 3 Isaac Newton Institute of Chile, Armenian Branch, 0213 Byurakan, Aragatsotn province, Armenia \\ ${ }^{4}$ Centro de Astrofísica da Universidade do Porto, Rua das Estrelas, 4150-762 Porto, Portugal \\ 5 Institut d'Astrophysique de Paris, UMR 7095 CNRS - UPMC, 98bis Bd Arago, 75014 Paris, France \\ 6 INAF - Osservatorio Astronomico di Padova, Vicolo dell'Osservatorio 5, 35122 Padova, Italy
}

Received 4 May 2012 / Accepted 20 June 2012

\section{ABSTRACT}

\begin{abstract}
Context. In this first paper of a series, we report the creation of large and well-defined database that combines extensive new measurements and a literature search of 3876 supernovae (SNe) and their 3679 host galaxies located in the sky area covered by the Sloan Digital Sky Survey (SDSS) Data Release 8 (DR8).

Aims. This database should be much larger than previous ones, and should contain a homogenous set of global parameters of SN hosts, including morphological classifications and measures of nuclear activity.

Methods. The measurements of apparent magnitudes, diameters $\left(D_{25}\right)$, axial ratios $(b / a)$, and position angles (PA) of SN host galaxies were made using the images extracted from the SDSS $g$-band. For each host galaxy, we analyzed RGB images of the SDSS to accurately measure the position of its nucleus to provide the SDSS name. With these images, we also provide the host galaxy's morphological type, and note if it has a bar, a disturbed disk, and whether it is part of an interacting or merging system. In addition, the SDSS nuclear spectra were analyzed to diagnose the central power source of the galaxies. Special attention was paid to collect accurate data on the spectroscopic classes, coordinates, offsets of SNe, and heliocentric redshifts of the host galaxies.

Results. Identification of the host galaxy sample is $91 \%$ complete (with $3536 \mathrm{SNe}$ in 3340 hosts), of which the SDSS names of $\sim 1100$ anonymous hosts are listed for the first time. The morphological classification is available for 2104 host galaxies, including 73 (56) hosts in interacting (merging) systems. The total sample of host galaxies collects heliocentric redshifts for 3317 ( $\sim 90 \%$ ) galaxies. The $g$-band magnitudes, $D_{25}, b / a$, and PA are available for 2030 hosts of the morphologically classified sample of galaxies. Nuclear activity measures are provided for 1189 host galaxies. We analyze and discuss many selection effects and biases that can significantly affect any future analysis of our sample.

Conclusions. The creation of this large database will help to better understand how the different types of SNe are correlated with the properties of the nuclei and global physical parameters of the host galaxies, and minimize possible selection effects and errors that often arise when data are selected from different sources and catalogs.
\end{abstract}

Key words. astronomical databases: miscellaneous - supernovae: general - galaxies: general - galaxies: fundamental parameters galaxies: structure

\section{Introduction}

A crucial aspect of many recent studies of extragalactic supernovae $(\mathrm{SNe})$ is to establish the links between the nature of SN progenitors and stellar populations of their host galaxies. The most direct method for realizing this is through their identification on pre-SN images. However, the number of such $\mathrm{SNe}$ is small and is limited to the nearby core-collapse (CC) events (e.g., Smartt 2009). This limitation and small-number statistics are the main reasons to investigate the properties of SN progenitors through indirect methods. In this context, the properties of SN host galaxies, such as the morphology, color, nuclear activity, star formation rate, metallicity, stellar population, age etc. provide strong clues to the understanding of the progenitors

* Full Table 7 is only available at the CDS via anonymous ftp to cdsarc.u-strasbg.fr (130.79.128.5) or via

http://cdsarc.u-strasbg.fr/cgi-bin/qcat?]/A+A/544/A81 (e.g., Petrosian \& Turatto 1995; Kazarian 1997; Bressan et al. 2002; Prantzos \& Boissier 2003; Mannucci et al. 2005; Prieto et al. 2008; Boissier \& Prantzos 2009; Arcavi et al. 2010; Han et al. 2010; Kelly \& Kirshner 2011). In addition, valuable information of the nature of progenitors can be obtained through the study of the spatial distribution of SNe (e.g., Förster \& Schawinski 2008; Hakobyan et al. 2009; Wang et al. 2010) and environments (e.g., Anderson \& James 2008; Modjaz et al. 2011; Leloudas et al. 2011).

Over the past decade, many studies have investigated the nature of SN progenitors in the nearby Universe via local and global properties of their host galaxies. For example, Prieto et al. (2008) investigated how the different types of SNe are correlated with the metallicity of their host galaxy. They showed strong evidence that $\mathrm{SNe} \mathrm{Ibc}^{1}$ occur in higher metallicity hosts than $\mathrm{SNe}$ II,

1 We use SNe Ibc to mainly denote the Ib, Ic and mixed Ib/c SNe types whose specific Ib or Ic classification is uncertain. 
while there is no such effect for SNe Ia relative to SNe II. Förster $\&$ Schawinski (2008) studied the radial distribution of SNe Ia in morphologically selected early-type host galaxies from the SDSS, and found that there is no statistically significant difference between the radial distribution of SNe Ia and the light profile of their early-type host galaxies. Hakobyan et al. (2009) compared the radial distribution of $\mathrm{CC}$ SNe within their spiral hosts with the distributions of stars and ionized gas in spiral disks. They concluded that the normalized radial distribution of all CC SNe is consistent with an exponential law, the scale length of the distribution of SNe II appears to be significantly larger than that of the stellar disks of their host galaxies, while SNe Ibc have a significantly smaller scale length than SNe II (see also Anderson \& James 2009). The scale length of the radial distribution of CC SNe shows no significant correlation with the host galaxy morphological type, or the presence of bar structure.

Several authors have studied the radial distributions of $\mathrm{SNe}$ of different types in large numbers of galaxies (e.g., Iye \& Kodaira 1975; Bartunov et al. 1992; van den Bergh 1997; Förster \& Schawinski 2008; Hakobyan et al. 2009), but none of these studies attempted to categorize the hosts according to their activity level. However, other authors have shown that the SNe distributions in galaxies with various activity levels might be different (e.g., Petrosian \& Turatto 1990; Petrosian et al. 2005; Hakobyan 2008; Wang et al. 2010; Herrero-Illana et al. 2012). For example, Wang et al. (2010) directly measured number and surface density distributions of SNe II in their hosts, and indicated that SNe II detected in star-forming galaxies follow an exponential law, in contrast, the distribution of SNe II detected in Active Galactic Nuclei (AGN) hosts significantly deviates from an exponential law. Petrosian et al. (2005) studying a sample of CC SNe in galaxies hosting AGN found that SNe Ibc in active/star-forming galaxies are more centrally concentrated than are the SNe II, but given the small sample, this difference was not statistically significant. The results of Petrosian et al. were confirmed with larger samples of CC SNe by Hakobyan (2008).

The locations of SN explosions in multiple galaxy systems have also been studied. In interacting galaxies, $\mathrm{CC} \mathrm{SNe}$ are not preferentially located toward the companion galaxy (e.g., Navasardyan et al. 2001). Similarly, the azimuthal distributions inside the host members of galaxy groups are consistent with being isotropic (Navasardyan et al. 2001). Han et al. (2010) found that $\mathrm{SNe}$ Ia are more likely to occur in isolated galaxies without close neighbors.

However, many similar studies (e.g., Anderson \& James 2009; Hakobyan et al. 2009; Wang et al. 2010) presented above are suffered from poor statistics, as well as strong biases on the SNe and their host galaxies sample. Often, they were random selections of nearby $\mathrm{SNe}$ and their hosts from the Asiago Supernova Catalogue ${ }^{2}$ (ASC, Barbon et al. 1999) or the Sternberg Astronomical Institute (SAI) Supernova Catalogue ${ }^{3}$ (SSC, Tsvetkov et al. 2004) or the official list of all the discovered SNe on the Central Bureau for Astronomical Telegrams (CBAT) website 4 . Most recently, Lennarz et al. (2012) published a unified SN catalog for 5526 extragalactic SN that were discovered up to 2010 December 31 . The unified catalog mostly combines ASC, SSC, and data from CBAT in a consistent way, and adopts all of the inhomogeneous data on $\mathrm{SNe}$ and their

\footnotetext{
2 http://web.oapd.inaf.it/supern/cat/

3 http://www.sai.msu.su/sn/sncat/

4 http://www . cbat . eps . harvard.edu/lists/Supernovae. html
}

host galaxies from the original sources. For the galaxy data, these catalogs made large use of the Third Reference Catalogue of Bright Galaxies (RC3) by de Vaucouleurs et al. (1991) and the HyperLeda ${ }^{5}$ database (Paturel et al. 2003) as well as the NASA/IPAC Extragalactic Database ${ }^{6}$ (NED). Hence, the data are given with various degrees of accuracy depending on the accuracy of the original catalog. Many selection effects and errors that often arise when data is selected from different sources and catalogs can significantly bias results and lead to wrong conclusions. Quantitative studies of SN progenitors therefore require a large and well-defined sample of SNe and their host galaxies, and our goal is to provide the database for such a sample.

The Eighth Data Release 7 (DR8) of the SDSS (Aihara et al. 2011) covering over 14000 square degrees with high quality imaging and spectroscopy makes it finally possible to construct better samples for studies of the properties of the host galaxies and environments of SNe. This large amount of the SDSS data enables statistically meaningful studies that are only little affected by selection effects. In this paper, we report the creation of a large database of several thousand $\mathrm{SNe}$ that exploded in galaxies identified in the SDSS DR8. We provide identifications of SN host galaxies, their accurate coordinates, heliocentric redshifts, morphological types, and activity classes, as well as apparent magnitudes, diameters, axial ratios, and position angles. However, our goal is not just to increase the size of the sample in comparison with previous studies, but also to carry out morphological classification, as well as individual measurements of the global parameters of the host galaxies in a homogenous way. In addition, we summarize the overall statistical properties of the sample, analyzing and discussing residual selection effects and biases that can still affect subsequent studies and results.

An additional motivation for this study is the comparison between the ASC, SSC, and CBAT databases, to reveal possible inconsistencies in the listed SNe types and offsets. Our database includes corrected data with their uncertainties on the SNe.

This is the first paper of a series and is organized as follows: Sect. 2 introduces the data and describes in detail the reduction techniques. In Sect. 3, we give the results and discuss all the statistical properties and selection effects of the sample. A summary and perspectives for future uses are finally addressed in Sect. 4. Throughout this paper, we adopt a cosmological model with $\Omega_{\mathrm{m}}=0.27, \Omega_{\Lambda}=0.73$, and the Hubble constant of $H_{0}=73 \mathrm{~km} \mathrm{~s}^{-1} \mathrm{Mpc}^{-1}$ (Spergel et al. 2007), consistent with direct determination based on Cepheid variables and $\mathrm{SNe}$ Ia by Riess et al. (2009).

Future papers of this series will use this database to determine how the different types of SN progenitors are correlated with the global parameters (morphology, size, luminosities etc.) of the host galaxies as well as on their nuclear properties (activity class, metallicity, stellar population etc.).

\section{Data and reduction}

\subsection{Supernova catalogs}

The ASC and SSC are compilations of information about SN discoveries obtained mainly from reports in the International Astronomical Union (IAU) Circulars and CBAT, as well as basic information about the host galaxies generally from the RC3, HyperLeda, and NED. We used the ASC, updated on

\footnotetext{
http://leda.univ-lyon $1 . \mathrm{fr} /$

6 http://ned.ipac.caltech.edu/

7 http://www. sdss3.org/
} 
Table 1. Distribution of main SN types in the SN catalogs.

\begin{tabular}{lcccccc}
\hline \hline Catalog & $\begin{array}{c}\text { Unclassified } \\
\%\end{array}$ & $\begin{array}{c}\text { I } \\
\%\end{array}$ & $\begin{array}{c}\text { Ia } \\
\%\end{array}$ & $\begin{array}{c}\text { Ibc } \\
\%\end{array}$ & $\begin{array}{c}\text { II } \\
\%\end{array}$ & $\begin{array}{c}\text { All } \\
N\end{array}$ \\
\hline ASC & 19.8 & 1.5 & 47.0 & 6.9 & 24.8 & 5609 \\
SSC & 21.1 & 2.2 & 46.1 & 6.8 & 23.8 & 5539 \\
\hline
\end{tabular}

March 2011, and SSC, updated on February 2011, to obtain and investigate general properties of $\mathrm{SNe}$ (designation, coordinates, offset, type etc.) and their host galaxies (name, coordinates, morphology, heliocentric redshift etc.). Table 1 displays the distribution of different types of $\mathrm{SNe}$ in updated versions of the ASC and SSC. Note that SNe II include subtypes II P (Plateau), II L (Linear), IIn (narrow-line), and IIb (transitional), but these subtypes are often absent in the SN catalogs.

We cross-matched the SN catalogs with the SDSS DR8 galaxies, mainly using, for the former, the coordinates of the $\mathrm{SNe}$, or of their host galaxies when the $\mathrm{SNe}$ positions were unavailable.

\subsection{The Sloan Digital Sky Survey}

The SDSS is a large photometric and spectroscopic survey of the Northern sky (mainly), using a dedicated $2.5 \mathrm{~m}$ telescope with a wide field of view $\left(3^{\circ}\right)$ at the Apache Point, New Mexico (e.g., Gunn et al. 2006). The first, second, and third phases of the SDSS (SDSS-I, SDSS-II, and SDSS-III) have produced eight data releases (see Aihara et al. 2011). The DR8, made publicly available in January 2011, contains all of the imaging data taken by the SDSS imaging camera (14555 square degrees of sky), including two large contiguous zones, one around the Northern Galactic Cap and one close to the Southern Galactic Cap (centered on RA $\sim 0^{\mathrm{h}}$ ). The DR8 also contains spectra from the SDSS spectrograph in an area covering 9274 square degrees. The photometric survey uses a specially designed multiband CCD camera that covers five bands over a wide wavelength range denoted by $u, g, r, i$, and $z$ with effective wavelengths of $3551 \AA, 4686 \AA$, $6165 \AA, 7481 \AA$, and $8931 \AA$, respectively. All of the imaging data have been reduced using improved data processing pipelines (for more details see Aihara et al. 2011). The SDSS spectra are observed using a pair of fiber-fed double spectrographs. Each of the two spectrographs collect spectra on 2 SITe/Tektronix 2048 by 2048 CCDs, one covering a wavelength range of $3800 \AA$ to $6100 \AA$, and the other from $5900 \AA$ to $9100 \AA$. The resolving power $(\lambda / \Delta \lambda)$ of the SDSS spectra ranges from 1800 to 2200 (Uomoto et al. 1999).

Currently, the total spectroscopic sample of the SDSS DR8 consists of 1629129 unique spectra and also 214071 repeated measurements. The spectra are classified and divided into different categories. The total galaxy sample of DR8 collects 860836 spectra and the SDSS Main Galaxy spectroscopic sample consists of 711726 objects with Petrosian magnitudes (corrected for Galactic extinction) $r \leq 17.77$ (Strauss et al. 2002). Below, the comparisons of physical parameters of the $\mathrm{SNe}$ host galaxy sample are performed with the SDSS Main Galaxy sample.

\subsection{Cross-matching the SNe with the SDSS galaxies}

The number of SNe from the aforementioned SN catalogs that matched with the SDSS DR8 is 3841 (in 3645 host galaxies). In several cases, the galaxies do not fully lie in the frame (field) of the DR8, but are fully covered in the DR7 fields. Also there are

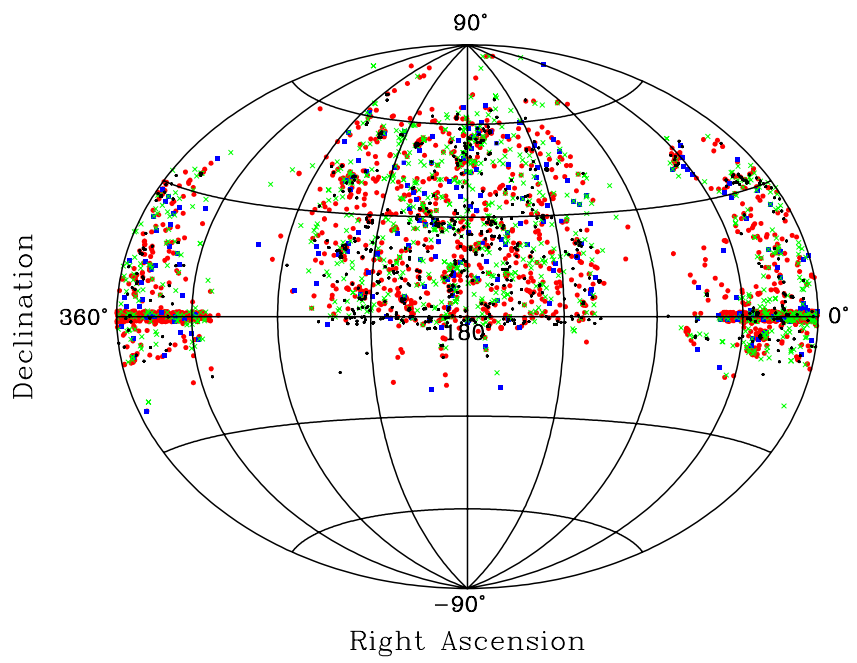

Fig. 1. The Aitoff projection of equatorial coordinates of $\mathrm{SNe}$ from the total sample. The symbols correspond to different types of SNe: Ia (red filled circles), Ibc (blue filled squares), II (green crosses), I and unclassified $\mathrm{SNe}$ (black dots).

cases when galaxies are in the DR8 field but with empty (black) images, while they are available in DR7. We therefore also crossmatched the SN catalogs with the SDSS DR7 and found an additional $35 \mathrm{SNe}$ in 34 host galaxies. In total, the sample of SNe contains 3876 events in 3679 host galaxies. The total sample includes 163 host galaxies with multiple SNe: 2, 3, 4, 5 and $6 \mathrm{SNe}$ are found in $135,19,5,1$ and 1 galaxy, respectively.

The distribution of equatorial coordinates of different types of SNe from the total sample is shown in Fig. 1. The distribution reflects the sky coverage of the final SDSS imaging survey, and different observational biases for different regions of the sky. Relatively empty regions in the northern sky are due to obscuration by the Galactic Plane. The SNe density peak around the celestial equator is caused by various $\mathrm{SN}$ surveys such as the SDSS SN Survey (Frieman et al. 2008) and ESSENCE (Miknaitis et al. 2007). These surveys discovered many targets, but because their main focus are on high-redshift SN Ia, most of their spectroscopically classified $\mathrm{SNe}$ are Ia (red filled circles), although roughly $100(\approx 10 \%)$ are CC SNe (blue filled squares and green crosses).

We visually inspected all images of our 3679 cross-matched SN host galaxies from the SDSS Imaging Server ${ }^{8}$, which builds RGB color images from the $g, r$, and $i$ data channels. This has been done to directly identify the hosts and to exclude the galaxies that were wrongly selected as hosts in the SN catalogs. All the identified hosts are the closest galaxies to the SN in terms of angular separation and if available also redshift. We excluded SN-host/SDSS matches when a SN was closer in angular separation and further in redshift (when available). We have also performed an extensive literature search mainly through the NED and HyperLeda databases, as well as the CBAT website, so as to find any additional data on their identification as SN host galaxies. For example, many original sources such as IAUC, CBAT, and unique papers include data on host galaxy identification, names, morphology, and description of many details (spiral arms, nucleus, tidal bridges, peculiarities etc.) as well as SNe magnitudes in different bands at different epochs, redshifts and many other information.

\footnotetext{
8 http://skyserver.sdss3.org/dr8/en/tools/chart/list. asp
} 
We identified $\sim 91 \%$ (3340 hosts with a total of $3536 \mathrm{SNe}$ ) of SN host galaxies that matched with the SDSS. Nine host galaxies with a total of $10 \mathrm{SNe}$ were not identified because of a few unavailable RGB images. The remaining 330 hosts mostly were unidentified due to insufficient resolution of SDSS images at the large distance of these objects. Among the identified hosts, roughly 2000 in the ASC ( 1100 in the SSC) are marked as anonymous. For these galaxies, we provide the SDSS names based upon the SDSS fiber targeted at the galaxy nucleus. In addition to the SDSS names, we used the multi-band images of the hosts and their appropriate photometric and spectral data to provide their accurate (J2000) equatorial coordinates, apparent $g$-band magnitudes, major axes $\left(D_{25}\right)$, axial ratios $(b / a)$, position angles (PA), morphological types, heliocentric redshifts, and nuclear activity classes.

\subsection{Spectroscopic classes of supernovae}

The spectral type of an SN provides a crucial diagnostic of the initial mass, age and metallicity of the progenitor, as well as of the explosion mechanism (e.g., Turatto 2003; Turatto et al. 2007). We have thus attempted to homogenize the spectral types for SNe that have been assign different types in different SN catalogs. In particular, all the SN types taken from the ASC and SSC were compared. When the types of given $\mathrm{SNe}$ did not match in these two catalogs, a comprehensive search of the literature was performed. This search was important because primary SN classifications can be changed with more accurate subsequent observations.

As an example, SN 1972R is listed as type Ib: (uncertain type Ib) and IPec (peculiar type I), in the ASC and SSC, respectively. Following claims regarding possible errors in the photometry of SNe carried out at Asiago observatory during the 70's, Patat et al. (1997) presented the result of new photometry of SNe Ia. They reported SN 1972R as type Ia observed at Asiago, not included in their sample but re-calibrated by Tsvetkov. As another example, in the ASC, the spectroscopic type of SN 2005az is listed as Ib. The Nearby Supernova Factory reported that a spectrum of SN 2005az, obtained, 5 days after its discovery, with the Integral Field Spectrograph on the University of Hawaii $2.2 \mathrm{~m}$ telescope, shows the SN to be a type Ib (Aldering et al. 2005). At the same time, in the SSC, according to Quimby et al. (2005), this SN is listed as type Ic. The SN was discovered approximately 17 days before maximum and spectroscopically classified 3 days after discovery as a SN Ic (Quimby et al. 2005). Finally, Kelly \& Kirshner (2011) updated the classification using a comprehensive set of spectra and confirmed that it was a type Ic explosion. We have adopted this latter type, for this SN, in our database.

In addition, to observe the spectroscopic transition that is typical for SN IIb, spectra taken over several epochs are required, but such data is not always included in the SN catalogs. For instance, SN 2006dj is listed in the ASC as type Ib, while the SSC and CBAT website list it as a transitional type IIb. Another example is SN 2002au, which is typed as Ia: (uncertain type Ia) in the SN catalogs. The spectroscopic type mentioned in Filippenko \& Chornock (2002) is a probable type Ia. Recently, Leaman et al. (2011) analyzed the spectrum observed by Filippenko \& Chornock, and suggested type IIb SN, which is what we adopt in our database.

SNe 1993R (Filippenko \& Matheson 1993), 2007jc (Pojmanski 2007), 2007kr (Bassett et al. 2007a), and 2007me (Bassett et al. 2007b) have shown spectral properties of type Ia as well as type Ic. For these $\mathrm{SNe}$, we have adopted a peculiar type I (I pec) instead of types Ia/c that are listed in the ASC. In addition, for SN 1997ew (Nugent et al. 1998), we adopted a peculiar type II (II pec) instead of type II/Ic that is given in the ASC.

Our sample of SNe includes also a small number of type $\mathrm{Ib}$ SNe (2000ds, 2001co, 2003dg, 2003dr, 2005E, and 2007ke) that are calcium-rich and may have different progenitors from typical SN Ib (e.g., Perets et al. 2010; Kawabata et al. 2010). However, the nature of this subtype of SNe is still under debate (e.g., Perets et al. 2011; Suh et al. 2011) and therefore, in our study, we haven't separated these SNe into a separate class. In addition, the extreme objects described in Pastorello (2012) have not been considered throughout this paper because of their rarity.

We did not include in our sample SN 1984Z, because it is an unconfirmed SN and is missing from the SSC and CBAT. SN $1989 Z$ is probably a foreground variable star (Bettoni et al. 1998; Kushida et al. 1998), and SN 2010U was a luminous fast nova (Humphreys et al. 2010). These two objects were also excluded from our database. SNe 1954J (Smith et al. 2010), 1997bs (van Dyk et al. 2000), 1999bw (Filippenko et al. 1999), 2000ch (Wagner et al. 2004), 2001ac (Matheson et al. 2001), 2002bu (Smith et al. 2011), 2002kg (Maund et al. 2006), 2003gm (Patat et al. 2003), and 2006bv (Smith et al. 2011) have been shown to actually be outbursts of Luminous Blue Variables (LBVs) similar to $\eta$-Carinae or $P$-Cygni, so we have omitted them from our database.

In addition, our $\mathrm{SNe}$ sample includes $62 \mathrm{SNe}$ discovered with the QUasar Equatorial Survey Team (QUEST). By analyzing the available SDSS spectra of these SNe, we found that SNe 2001 ap and 2001 at (Schaefer 2001) are indeed foreground A0 type stars, while SN 2001aw (Schaefer 2001) is a foreground cataclysmic variable. We removed these 3 objects from our database too, since they are not true SN explosions.

During the mutual comparison between the SN catalogs, as well as in our literature search, we have updated spectroscopic types for $67 \mathrm{SNe}$. All the updated SN classifications are labeled by the letter U. We collected all the available data on $3166 \mathrm{SNe}$ types when they were available in one of the SN catalogs or in the CBAT. Our total sample consists of 72 SNe I, 1990 SNe Ia, 234 SNe Ibc, 870 SNe II (including II P, II L, IIn, and IIb), and 710 unclassified SNe. All the uncertain (“:” or "“?”) and peculiar ("pec") classifications are flagged. Types I, Ia, and II include also a few SNe classified from the light curve only: these SN types are labeled by “*” symbols.

\subsection{Supernova offsets}

The locations of SNe within host galaxies, regardless of their morphological types, provide a powerful clue toward distinguishing SN progenitor scenarios (e.g., Bartunov et al. 1994; van Dyk et al. 1996; Tsvetkov et al. 2001; Anderson \& James 2008; Hakobyan et al. 2009; Modjaz et al. 2011; Leloudas et al. 2011). The SN location is usually provided by the SN catalogs via its offset from the host galaxy nucleus, in the East/West (E/W) and North/South (N/S) directions, in arcsec (e.g., Barbon et al. 1999; Tsvetkov et al. 2004). Ideally, the equatorial coordinates of an SN can be easily derived from the coordinates of its host galaxy and its offset. Alternatively, SN offsets $(\Delta \alpha, \Delta \delta)$ can be simply calculated by $\Delta \alpha \approx\left(\alpha_{\mathrm{SN}}-\alpha_{\mathrm{g}}\right) \cos \delta_{\mathrm{g}}$ and $\Delta \delta \approx\left(\delta_{\mathrm{SN}}-\delta_{\mathrm{g}}\right)$, where $\alpha_{\mathrm{SN}}$ and $\delta_{\mathrm{SN}}$ are the $\mathrm{SN}$ coordinates, $\alpha_{\mathrm{g}}$ and $\delta_{\mathrm{g}}$ are the host galaxy coordinates in equatorial system. However, the astrometric data given in the SN catalogs have various degrees of accuracy depending on many factors. Below we 
present and discuss several examples, when there are contradictions in SN offsets among different sources.

In the ASC, the coordinates of SN $1987 \mathrm{~F}$ are $\alpha_{\mathrm{SN}}=$ $12^{\mathrm{h}} 41^{\mathrm{m}} 38.99, \delta_{\mathrm{SN}}=+26^{\circ} 04^{\prime} 22^{\prime \prime} .4(\mathrm{~J} 2000.0)^{9}$, and the $\mathrm{SN}$ is located at 20" East and 5" South from the nucleus of PGC 042584 (NGC 4615). Originally, this SN was discovered on March 22 at about 20" East of the nucleus of host galaxy (Cherepashchuk et al. 1987). On April 23, the object was independently rediscovered at 24" East and 6" South of the host nucleus (Wild \& Schildknecht 1987). The latter offset is given in the SSC. Using the SDSS images, we identified the nucleus of PGC 042584 at $\alpha_{\mathrm{g}}=12^{\mathrm{h}} 41^{\mathrm{m}} 37^{\mathrm{s}} .31, \delta_{\mathrm{g}}=+26^{\circ} 04^{\prime} 22^{\prime \prime} .1$. Given the absolute positions of the $\mathrm{SN}$ and the nucleus of its host galaxy, we deduce the offset of SN 1987F to be 22'.' 6 East and 0.'3 North. This leads to a $5^{\prime \prime}$ difference in the $\Delta \delta$ component of the SN offset from that listed in the SN catalogs.

The ASC lists the position of SN 2001en at $\alpha_{\mathrm{SN}}=$ $01^{\mathrm{h}} 25^{\mathrm{m}} 22^{\mathrm{s}} .90, \delta_{\mathrm{SN}}=+34^{\circ} 01^{\prime} 30^{\prime} .5$, while, according to Hutchings \& Li (2001), it is located 6". 4 East and 2".8 South from the eastern nucleus of PGC 005268 (NGC 523). The latter offset is given also in the SSC. The SDSS image of this galaxy (see Fig. 3) suggests that it has a peculiar structure, and the physical center of PGC 005268, from which the spiral arms begin, is located at $\alpha_{\mathrm{g}}=01^{\mathrm{h}} 25^{\mathrm{m}} 20^{\mathrm{s}} .75, \delta_{\mathrm{g}}=+34^{\circ} 01^{\prime} 29^{\prime}$. 8 . Taking into account both the coordinates of the SN and its host galaxy, as well as definition of SN offset as galactocentric distance in the corresponding directions (Barbon et al. 1999; Tsvetkov et al. 2004), the offset for SN 2001en should be corrected to 26".7 East and 0.7 North. This means that by not taking the center of the host galaxy as a starting point for the offset calculation, a difference in the $\Delta \alpha$ component of the $\mathrm{SN}$ real offset is about $20^{\prime \prime}$, comparing to that reported in the $\mathrm{SN}$ catalogs.

In the ASC, the coordinates of SN 2010br are $\alpha_{\mathrm{SN}}=$ $12^{\mathrm{h}} 03^{\mathrm{m}} 10^{\mathrm{s}} 95, \delta_{\mathrm{SN}}=+44^{\circ} 31^{\prime} 43^{\prime \prime} .1$, while its offset is about 19.'5 East and 10" South (Maksym et al. 2010) from the nucleus of PGC 038068 (NGC 4051). The SSC lists the coordinates of the $\mathrm{SN}$ as $\alpha_{\mathrm{SN}}=12^{\mathrm{h}} 03^{\mathrm{m}} 10^{\mathrm{s}} .96, \delta_{\mathrm{SN}}=+44^{\circ} 31^{\prime} 42^{\prime \prime} \cdot 9$ and offset as 14.'3 East and 9.'7 South (Maxwell et al. 2010). We measure the accurate coordinates of the nucleus of PGC 038068 to be $\alpha_{\mathrm{g}}=12^{\mathrm{h}} 03^{\mathrm{m}} 09^{\mathrm{s}} .61, \delta_{\mathrm{g}}=+44^{\circ} 31^{\prime} 52^{\prime \prime}$. 6 , yielding an offset of $14^{\prime \prime} 3$ East and 9.'5 South, which is consistent with that reported in the SSC (Maxwell et al. 2010), but different in the $\Delta \alpha$ component of about $5^{\prime \prime}$ from that reported in the ASC.

The ASC collects 2352 offsets, and 3179 coordinates for $\mathrm{SNe}$ in the sample of identified host galaxies. At the same time, for these SNe, the SSC includes 2285 offsets, and 3496 coordinates. In total, $150 \mathrm{SNe}$ have discrepancies in the offset components $\Delta \alpha$ or $\Delta \delta$ greater than $4^{\prime \prime}$ from those that we derived from our accurate measurements of the coordinates of the $\mathrm{SNe}$ and their host galaxy nuclei. The total sample also includes $340 \mathrm{SNe}$ with unidentified hosts in the SDSS. For these SNe, the two catalogs collect 91 offsets and 339 coordinates. Among all $\mathrm{SNe}$ of the total sample, 40 show offset discrepancies of more than $4^{\prime \prime}$, in at least one component, between the ASC and SSC values.

These examples of inconsistency of SNe offsets clearly show that the information in the SN catalogs is not always correct, which can affect any statistical study based on the use of offsets. For this reason, we have conducted a wide search of SNe offset data in the literature (mostly CBAT and IAU Circulars) for all cases where discrepancies greater than $4^{\prime \prime}$ were found in $\Delta \alpha$ or $\Delta \delta$. Our aims were to find correct offsets and to flag the cases where well-defined data could not be determined. In general,

\footnotetext{
9 We always adopt J2000.0 as the system of equatorial coordinates.
}

the ASC offsets are more reliable than the SSC ones, so offsets from the ASC were mostly adopted if they agreed with the SSC within 4", otherwise we used our own measurements.

In the end, our database contains in total $3599 \mathrm{SNe}$ with offset data; 2419 SNe with available offsets, and 1180 SNe with offsets determined by us. During the investigation, we corrected the offsets of 43 SNe. We also flagged offsets as uncertain (":") for $90 \mathrm{SNe}$ that show a large dispersion in the offsets (in the ASC, $\mathrm{SSC}, \mathrm{CBAT}$ as well as in our determination). In addition, we calculated unavailable coordinates for $332 \mathrm{SNe}$, via correct offsets and accurate coordinates of identified nuclei of their host galaxies. We flagged these coordinates as uncertain (":"), because the precise determination of the position of the host galaxy nucleus is difficult and depends on many factors (e.g., color of image, plate saturation, galaxy peculiarity etc.). Moreover, the SN catalogs report different offsets with different levels of accuracy. This information should be considered when analyzing individual accurate locations of these $\mathrm{SNe}$ (e.g., possible associations with $\mathrm{H}$ II regions, spiral arms etc.).

\subsection{Host galaxy morphologies}

The galaxy classification methodology used in this paper is based on classification scheme used by Petrosian et al. (2007) and is applied on the SDSS DR7 and DR8 RGB images. We classified SN host galaxies using the modified Hubble sequence (E-E/SO-SO-S0/a-Sa-Sab-Sb-Sbc-Sc-Scd-Sd$\mathrm{Sdm}-\mathrm{Sm}-\mathrm{Im}$ ), at the same time using morphological information in the SN catalogs as well as HyperLeda, along with images for galaxies obtained with NED. For mostly high-redshift hosts, we classified galaxies as " $S$ " when it was not possible to distinguish between the various Hubble subclasses of lenticulars or spirals. Some classifications are noted by symbols of uncertainties adopted from the RC3 scheme. Symbol ":" indicates that the classification is doubtful, "pec" indicates that the galaxy is peculiar (presence of shells, tidal tails etc.), and finally "?" indicates that the classification is highly uncertain. In addition, the host galaxies in interacting or merging systems have been flagged as "inter" or "merg", respectively.

We classified 2104 host galaxies, corresponding to $63 \%$ of our sample of galaxies identified in the SDSS. The remaining 1236 objects were not classified because of their small angular sizes $\left(\leq 5^{\prime \prime}\right)$. In Table 2 , we present the distributions of morphological types and barred structure of the classified SN host galaxies. The sample also includes 73 hosts in interacting ("inter"), and 56 hosts in merging ("merg") systems.

In order to test our morphological classification and detection of bar structure, a set of 100 host galaxies is randomly selected from the classified sample and re-classified. We estimated our mean confidence, comparing re-classification with the earlier classification of the same galaxies. This step allows estimating relative biases in our classification. The chances of our failure to detect bars and misclassification greater than two morphological units are both roughly $2 \%$.

\subsection{Isophotal measurements of host galaxies}

As one of our goals is to conduct detailed studies of the $\mathrm{SNe}$ distribution in different types of hosts, we require a galaxy to have well-defined apparent magnitude, major axis $\left(D_{25}\right)$, axial ratio $(b / a)$, and position angle (PA). To measure these parameters on $g$-band images of SDSS DR8, $25 \mathrm{mag} \operatorname{arcsec}^{-2}$ 
Table 2. Distributions of morphological types and barred structure of classified host galaxies.

\begin{tabular}{lccc}
\hline \hline Morphological type & With bars & Without bars & All \\
\hline E & 0 & 69 & 69 \\
E/S0 & 0 & 45 & 45 \\
S0 & 6 & 68 & 74 \\
S0/a & 19 & 76 & 95 \\
Sa & 18 & 48 & 66 \\
Sab & 30 & 58 & 88 \\
Sb & 126 & 186 & 312 \\
Sbc & 105 & 236 & 341 \\
Sc & 88 & 297 & 385 \\
Scd & 23 & 74 & 97 \\
Sd & 60 & 33 & 93 \\
Sdm & 28 & 20 & 48 \\
Sm & 7 & 20 & 27 \\
Im & 0 & 27 & 27 \\
\hline All & 510 & 1257 & 1767 \\
\hline
\end{tabular}

Notes. Our sample includes also 337 "disk-like" galaxies with "S" classification, among which 12 show a barred structure.

isophotes were constructed using the Graphical Astronomy and Image Analysis ${ }^{10}$ (GAIA) tool.

GAIA is a highly interactive image display tool with the additional capability of being extensible to integrate other programs. Its photometry package, PHOTOM $^{11}$, provides a possibility to measure aperture photometry with a highly interactive environment for controlling the positions, sizes and orientations of circular and elliptical apertures.

Starting from the measured $25 \mathrm{mag} \operatorname{arcsec}^{-2}$ isophotes, elliptical apertures were centered on the $g$-band galaxy centroid position (obtained manually). Major axes $\left(D_{25}\right)$, axial ratios $(b / a)$, and position angles (PA) of the galaxies were then obtained via PHOTOM in GAIA. All the elliptical apertures were also checked on host galaxy image by eye to ensure that they were in good fit with them. Apparent magnitudes were calculated via the total flux within the elliptical aperture. During the photometric measurements we masked out bright projected/saturated stars. In addition, apparent magnitudes and major axes were corrected for Galactic and host galaxy internal extinction (Schlegel et al. 1998; Bottinelli et al. 1995, respectively).

The SDSS images of 6 objects were not used, because of the presence of bright stars and background galaxies projected inside the elliptical apertures. It was difficult to remove these objects from the images without leaving residuals. Also, 31 galaxies have such peculiar geometries that it was not possible to measure isophotal quantities using elliptical aperture techniques. Moreover, 37 host galaxies, often with large angular sizes, were fragmented into separate SDSS fields, and it was not possible to apply the deprojection technique on the full extent of these galaxies.

In total, we have measured $g$-band magnitudes, major axes $\left(D_{25}\right)$, axial ratios $(b / a)$, and position angles (PA) for 2030 galaxies among the 2104 morphologically classified ones $(96 \%)$.

We now consider possible K-corrections to the photometry, but for this we need to assess the distribution of redshifts. The

\footnotetext{
${ }^{10}$ GAIA is available for download as part of JAC Starlink Release at http://starlink. jach.hawaii.edu

${ }^{11}$ http://www.starlink.ac.uk/docs/sun45.htx/sun45.html
}

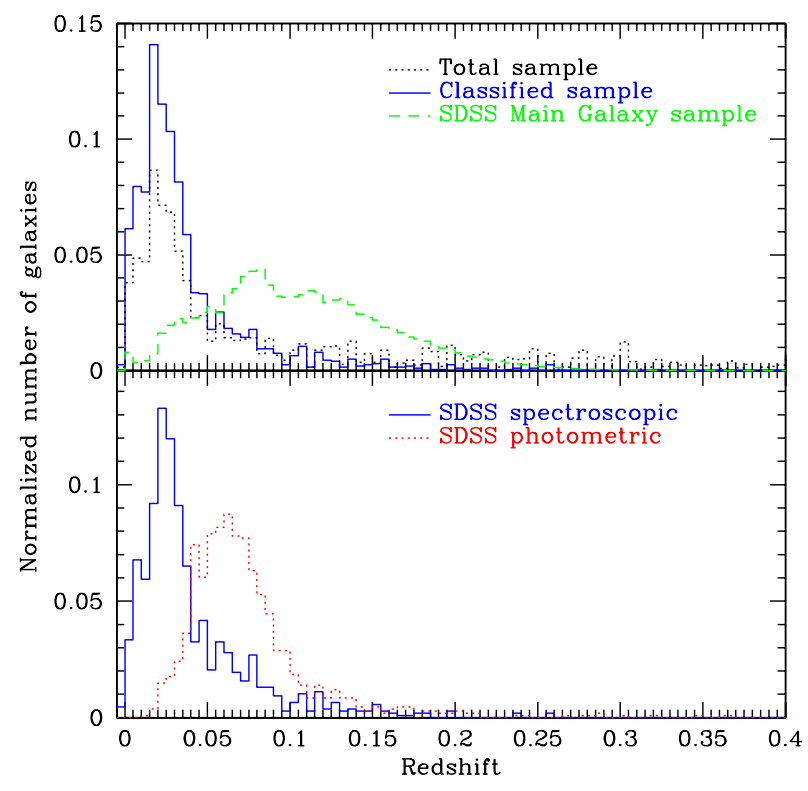

Fig. 2. Top: redshift distributions of galaxies in the total (black dotted), morphologically classified (blue solid), and SDSS Main Galaxy (green dashed) samples. Bottom: distribution of SDSS spectroscopic (blue solid) and photometric (red dotted) redshifts for classified host galaxies.

redshift completeness of the total sample of host galaxies is high: 3317 of 3679 hosts (90\%) have a redshift; 3232 redshifts were available in the SN catalogs, but for the 1214 galaxies that also have SDSS redshift measurements, we adopted the SDSS measurement. Moreover, SDSS provided redshifts for 85 galaxies that did not have redshifts in the SN catalogs. The redshift completeness of the morphologically classified sample is even higher: 2023 of 2104 hosts $(96 \%)$ have a redshift. The majority $(93 \%)$ of these host galaxies have $z \leq 0.1$. In the top panel of Fig. 2, we separately display the redshift distribution of host galaxies in the total and in morphologically classified samples. For comparison, the redshift distribution of $\sim 680000$ objects from the SDSS Main Galaxy sample is also shown.

Since the redshifts of classified galaxies are mostly low, their K-corrections are negligible and were ignored. However, we checked in the PhotoZ table of the SDSS database that the $\mathrm{K}$-corrections of these objects do not exceed $0.2 \mathrm{mag}$ in the $g$-band. We also compared the redshift distribution of galaxies, which simultaneously have photometric and spectroscopic redshifts in the SDSS. The photometric redshifts are less accurate than spectroscopic redshifts, and may be sufficient for understanding accuracy of estimation of the SDSS K-correction. The bottom panel of Fig. 2 clearly shows that photometric redshifts are overestimated on average by $\sim 0.03$, which suggests that aforementioned value $(0.2 \mathrm{mag})$ of the SDSS K-correction also is overestimated by $\sim 30 \%$.

To calculate the luminosity distances and absolute magnitudes of galaxies, we used the recession velocities both corrected to the centroid of the Local Group (Yahil et al. 1977), and for infall of the Local Group toward Virgo cluster (Theureau et al. 1998; Terry et al. 2002) $)^{12}$. For 48 nearby hosts out of 52 with $z \leq 0.003$, we were able to find distance estimates

\footnotetext{
12 We thus neglect the peculiar velocities of galaxies relative to the Virgo cluster. There could thus be non-negligible distance errors for galaxies closer than $z \approx 0.01$, which accounts for less than $4 \%$ of our total sample (see dashed histogram of top panel of Fig. 2).
} 


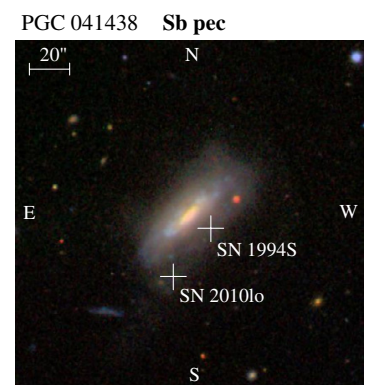

PGC 005268 SBc pec
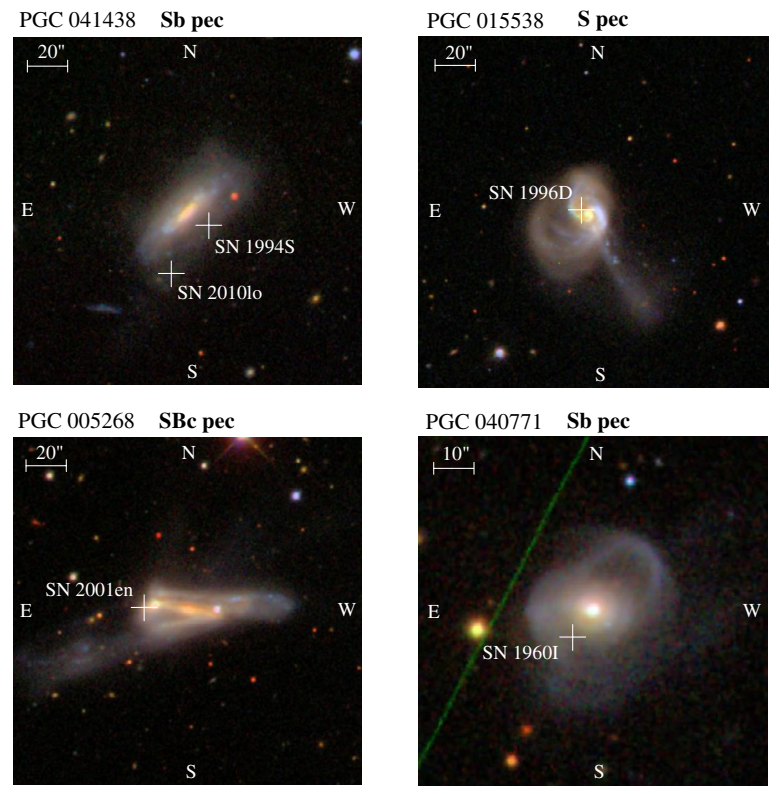

PGC 040771 Sb pec

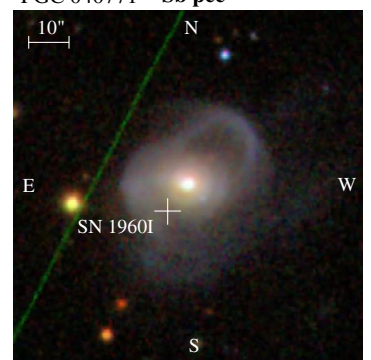

Fig. 3. Examples of SDSS DR8 images representing disturbed (diffused) or lopsided spiral disk of SN host galaxies. The PGC objects identifiers and our morphological classification are listed at the top. The SN names and positions (marked by a cross sign) are also shown. In all images, north is up and east to the left.

via redshift-independent distance indicators (e.g., derived from Cepheid variables or the Tully-Fisher relation) using NED and HyperLeda.

If spiral galaxies have disks that are not perfectly thin, then the measured axis ratio will not simply be the $b / a=\cos ^{-1} i$ for galaxies viewed with inclination $i$ relative to their polar axis. We calculate the inclinations of galaxies following the classical Hubble (1926) formula

$\sin ^{2} i=\frac{1-\operatorname{dex}[-2 \log (a / b)]}{1-\operatorname{dex}\left[-2 \log r_{0}\right]}$,

where $i$ is the inclination angle in degrees between the polar axis and the line of sight and $r_{0}$ is the intrinsic axis-ratio $a / b$ of galaxies viewed edge-on. According to Paturel et al. (1997), $\log \left(r_{0}\right)=0.43+0.053 t$ for $-5 \leq t \leq 7$ and $\log \left(r_{0}\right)=0.38$ for $t>7$, where $t$ is the de Vaucouleurs morphological type. We adopt $t=5$ for galaxies classified as " $\mathrm{S}$ " classification, because this is the most frequent type in other catalogs. This simple formula works well for most of disk galaxies, but probably not for Irregulars and Ellipticals (van den Bergh 1988).

Many CC SN studies are based on the assumption that these $\mathrm{SNe}$ have young progenitors that are located in the disks of spiral galaxies (e.g., van den Bergh 1997; Anderson \& James 2009; Hakobyan et al. 2009; Wang et al. 2010). The SN radial distances (e.g., Hakobyan et al. 2009) as well as metallicity gradients (e.g., Boissier \& Prantzos 2009) in disks are usually estimated from the de-projected separations from host galaxy nuclei, using the inclination correction. However, in many cases (clearly seen in 96 hosts), the galaxy disks are disturbed (diffused) or lopsided and far from the ideal disk structure, which makes the deprojection less secure. We therefore flagged these galaxies for specific investigations in future studies. In Fig. 3 we show examples of such flagged host galaxies.

\subsection{Nuclear activity levels of host galaxies}

The cross-matching of the SN catalogs with the SDSS DR7 and DR8 provides us with the spectra of the nuclei ${ }^{13}$ of SN host galaxies. We visually inspected the images from the SDSS Imaging Server to exclude the galaxies whose spectra were offset from their nuclei. Among the 3340 identified host galaxies, 1206 hosts (with $1287 \mathrm{SNe}$ ) have SDSS nuclear spectra ${ }^{14}$, which were analyzed to diagnose the central power source of the galaxies.

For each narrow emission-line galaxy, we have used the STARLIGHT spectral synthesis code (Cid Fernandes et al. 2004, 2005) to model the stellar spectral energy distribution (SED) for each SDSS spectra. The best-fitting stellar SED was then subtracted from the observed spectrum in order to isolate the pure emission line spectrum. This way, in many cases, even weak emission lines that seem to be absent in the observed spectra could be detected and measured accurately enough. STARLIGHT uses different techniques, combining empirical population synthesis and ingredients of evolutionary synthesis to compute the best-fitting stellar SED. The best-fitting linear combination of $N_{\star}$ Single Stellar Populations (SSPs), is obtained by using a non-uniform sampling of the parameter space based on the Markov Chain Monte Carlo method, plus an approach called simulated annealing, and a convergence criteria similar to that proposed by Gelman \& Rubin (1992), to approximately determine the global $\chi^{2}$ minimum.

For our analysis, we have chosen SSPs from Bruzual \& Charlot (2003), which are based on the "Padova 1994" evolutionary tracks (Alongi et al. 1993; Bressan et al. 1993; Fagotto et al. 1994a,b; Girardi et al. 1996) and the Chabrier (2003) Initial Mass Function (IMF) between 0.1 and $100 M_{\odot}$. The SSP library used here comprises six metallicities $(0.005,0.02,0.2,0.4,1$, and $2.5 Z_{\odot}$ ) for 25 ages between $1 \mathrm{Myr}$ and $18 \mathrm{Gyr}$. The intrinsic extinction has been modeled as a uniform dust screen, adopting the extinction law by Cardelli et al. (1989). Line broadening effects, due to line-of-sight stellar motions, are accounted for, in STARLIGHT, by Gaussian convolution.

We classified the spectra, according to enhanced starforming (SF) or AGN (Seyfert or LINER), using the $[\mathrm{O}$ III $] \lambda 5007 / \mathrm{H} \beta$ versus $[\mathrm{N} \mathrm{II}] \lambda 6583 / \mathrm{H} \alpha$ standard diagnostic diagram (Baldwin et al. 1981, hereafter BPT). We adopted the demarcation line proposed by Kauffmann et al. (2003, hereafter K03) to select SF hosts, and used the theoretical upper limit of SF galaxies proposed by Kewley et al. (2001, hereafter K01) to separate AGNs. The region between the two curves is occupied by the so-called composite objects (e.g., Kewley et al. 2006; Wang \& Wei 2008), whose spectra are believed to contain significant contributions from both SF and AGN. AGNs were separated by Seyfert-LINERs with the demarcation line proposed by Cid Fernandes et al. (2010 hereafter, C10).

We only consider host galaxies with high-quality spectra, i.e. with signal-to-noise ratio $(S / N)>5$ in the 4730-4780 $\AA$ continuum, and $S / N \geq 3$ in all emission-lines used in the BPT diagram. In addition, the SDSS spectra with bad flux calibrations or bad redshift determinations were also excluded. Finally, the hosts with broad emission lines ( $F W H M$ of $\mathrm{H} \alpha \geq 1000 \mathrm{~km} \mathrm{~s}^{-1}$ ), which were assigned as broad-line AGNs and galaxies without

\footnotetext{
13 The $3^{\prime \prime}$ fiber sees out to a projected radius of only $865 \mathrm{pc}$ for $z=0.03$ (the median of our sample) but as far as $2.7 \mathrm{kpc}$ for $z=0.1$. So, the spectra are not limited to the nuclei. Nevertheless, if there is nuclear activity, this should dominate the non-nuclear emission in the spectrum. ${ }^{14}$ Only 1214 galaxies in our sample have SDSS spectra, and 8 of these are not in the nucleus of the galaxy.
} 


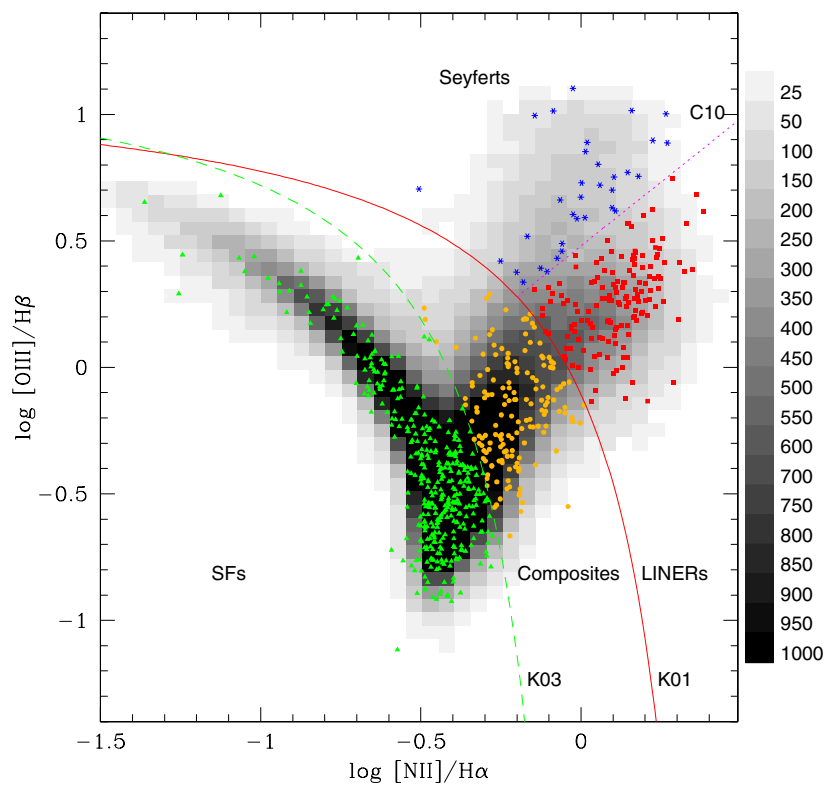

Fig. 4. BPT spectral diagnostic diagram for 727 host galaxies with narrow emission-lines. Star forming (SF) and composite galaxies are displayed with green triangles and orange circles, respectively. The blue asterisks and red squares respectively are Seyferts and LINERs. The red solid line shows the theoretical demarcation line separating AGN from SF galaxies proposed by Kewley et al. (2001, K01), while the green dashed line is the empirical line proposed by Kauffmann et al. (2003, K03). The magenta dotted line is the Seyfert-LINER demarcation line proposed by Cid Fernandes et al. (2010, C10). The underlying density distribution of the SDSS emission-line galaxies is shown in grayscale. The grayscale bar in the right represents the number of galaxies in each density bin.

any emission features were excluded from the BPT diagnostics but considered in studies of SN host galaxies. The distribution of 727 narrow emission-line galaxies in the BPT diagram is illustrated in Fig. 4. For comparison, the density distribution of the SDSS Main Galaxy sample ( 260000 emission-line objects), which passed our adopted $\mathrm{S} / \mathrm{N}$ criteria, is also shown.

However, our strong criteria on $\mathrm{S} / \mathrm{N}$ prevents us from including the weak emission-line galaxies. We therefore also used the WHAN diagram (Cid Fernandes et al. 2010, 2011): the equivalent width of $\mathrm{H} \alpha\left(W_{\mathrm{H} \alpha}\right)$ versus [N II] $\lambda 6583 / \mathrm{H} \alpha$ to diagnose the central power source of the hosts including those with weak emission lines. A simple transposition strategy was used to plot the K03 demarcation line in the WHAN diagram (Cid Fernandes et al. 2010). Here, AGNs were separated by the Seyfert-LINER demarcation line proposed by Kewley et al. (2006, K06). Also, the line, proposed by Cid Fernandes et al. (2011, C11), was used to separate retired/passive (RP) galaxies. This diagram allows us to plot nearly all $(92 \%)$ host galaxies with available spectra in the SDSS. The distribution of 1106 galaxies in the WHAN diagram is presented in Fig. 5.

In the BPT diagram, the hosts with SDSS nuclear spectra include 185 narrow-line AGN (33 Seyfert and 152 LINER), $382 \mathrm{SF}$, and 160 composite galaxies. The WHAN diagram includes in total 234 narrow-line AGN (151 Seyfert and 83 LINER), 568 SF, and 304 RP galaxies. As previously mentioned, we also have 6 broad-line AGNs and 77 passive (P) galaxies without any emission features. The activity of nucleus of 17 galaxies (with $21 \mathrm{SNe}$ ) could not be analyzed due to the poor-quality SDSS spectra.

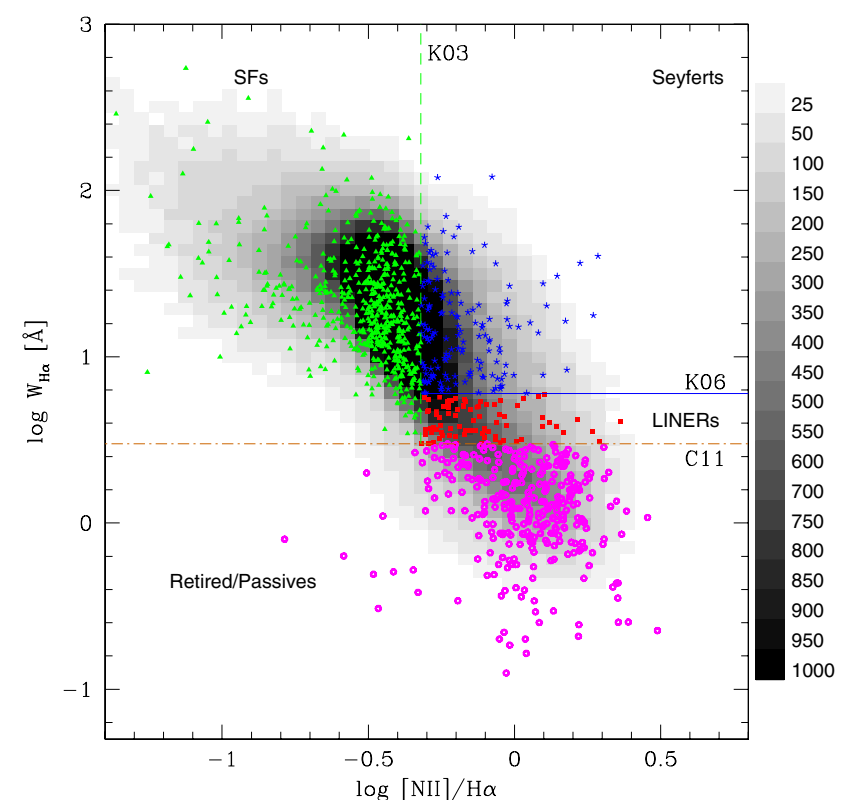

Fig. 5. WHAN spectral diagnostic diagram for 1106 host galaxies. SF galaxies are displayed with green triangles and separated by the K03 line. The blue asterisks and red squares respectively are Seyferts and LINERs, which are separated by blue solid line proposed by Kewley et al. (2006, K06). Retired/passive (RP) galaxies are marked by magenta circles and separated by the orange dashed-dotted line proposed by Cid Fernandes et al. (2011, C11). The underlying density distribution of the SDSS emission-line galaxies is shown in grayscale. The grayscale bar in the right represents the number of galaxies in each density bin.

\subsection{The total sample}

The first 27 (chronologically oldest SNe) and last 3 (most recent $\mathrm{SNe}$ ) entries of our total sample of SNe and their host galaxies are shown in Table 7 and the full table is available electronically. The full table contains 19 columns for $3876 \mathrm{SNe}$ (3679 host galaxies) and provides the following information: (Col. 1) designation of $\mathrm{SN}$; (Col. 2) right ascension of $\mathrm{SN}\left(\alpha_{\mathrm{SN}}\right)$ in degrees; (Col. 3) declination of SN ( $\left.\delta_{\mathrm{SN}}\right)$ in degrees; (Col. 4$)$ offset (in arcsec) of SN from the host galaxy nucleus; (Col. 5) spectroscopic type of SN; (Col. 6) SDSS identification of the host galaxy; (Col. 7) right ascension of host galaxy $\left(\alpha_{\mathrm{G}}\right)$ in degrees; $\left(\right.$ Col. 8) declination of host galaxy $\left(\delta_{\mathrm{G}}\right)$ in degrees; (Col. 9$)$ heliocentric redshift of the host; (Col. 10) morphological type of the host; (Col. 11) presence of bar in the host; (Col. 12) host in interacting ("inter") or in merging ("merg") systems; (Col. 13) host with disturbed disk structure; (Col. 14) measured major axis in arcsec (isophotal level of $25 \mathrm{mag} \operatorname{arcsec}^{-2}$ in the $g$-band) of the host; (Col. 15) axial ratio of the host; (Col. 16) position angle (in degrees) of the host; (Col. 17) measured apparent $g$-band magnitude; (Cols. 18 and 19) nuclear activity class of the host galaxy.

\section{Results and discussion}

In this section, we compare some of our measured parameters with previously available measurements, provide distributions and statistics on several of them, study their evolution with distance, and finally discuss various selection effects. This analysis is important to understand whether our sample of SN and their host galaxies from the SDSS DR8 is representative of the general population. 
Our comparisons with measurements from HyperLeda and SDSS are done by performing robust linear regressions, with iterative rejection of $3 \sigma$ outliers, and we also measure a robust estimate of the dispersion, using the median absolute deviation (MAD) of the residuals, converting to $\sigma_{\mathrm{MAD}}=1.483 \mathrm{MAD}$, where the numerical factor is the one appropriate for Gaussian distributions of residuals.

\subsection{Comparison of host galaxy morphological classifications}

It is well known that the central parts of the images of many very bright galaxies and high surface brightness objects may be over-exposed, which affects their morphological classification. Meanwhile, galaxies that are faint (low surface brightness) can also be misclassified, because of the lack of precise morphological details in the image.

Using a homogenous sample of $604 \mathrm{SNe}$, van den Bergh et al. (2002, 2003, 2005) classified the SN host galaxies from the Lick Observatory Supernova Search (LOSS) in the David Dunlap Observatory (DDO) morphological type system. They suggested that to understand the dependence of SN type on the host galaxy population, it is more important to obtain accurate morphological classifications than it is to increase the size of the sample. For example, among 800 morphologically classified hosts of CC SNe, Hakobyan et al. (2008) found 22 cases where the host had been classified as E or S0. Following a detailed morphological analysis, they found that among these 22 early-type objects, 17 are in fact misclassified spiral galaxies, one is a misclassified irregular, and one is a misclassified ring galaxy, leaving only 3 early-type galaxies ${ }^{15}$. In this respect, the host morphology is a crucial parameter in the study of $\mathrm{SN}$ progenitors.

To present a detailed numerical comparison of our morphological classification of SN host galaxies with those given in the $\mathrm{SN}$ catalogs (mainly from RC3), we introduce our $t$-type values that we will use in this study. In comparison to the standard RC3 classification, we have grouped Ellipticals and Lenticulars into broader classes: $\mathrm{cE}$ and $\mathrm{E}$ galaxies are typed together, as well as $\mathrm{S}^{-}, \mathrm{S}^{0}$ and $\mathrm{S}^{+}$galaxies. Indeed, using the SDSS images, it was not possible to visually distinguish the differences between these subclasses. Table 3 shows the relation between our $t$-types and those of the RC3.

In the top panel of Fig. 6, we show the comparison of our morphological classifications for the 1313 hosts from our classified sample that are also present in the HyperLeda database (when both classifications are more accurate than just "S": 1767 for our sample among the total of 2104 classified galaxies). The reference system of the morphological classification in HyperLeda is generally the RC3. The comparisons with $\mathrm{RC} 3$ types were performed after converting the RC3 numerical ( $t$ ) classifications to our scheme of $t$ versus type (see Table 3 ). Point sizes in the figure correspond to the number of hosts in each morphological bin. In the bottom panel of Fig. 6, we present the distributions of the differences between the HyperLeda $t$-types and ours.

Inspection of Fig. 6 shows a trend for our classifications to be later overall (except E) in comparison with those of HyperLeda. The mean deviation in classifications is $0.65 \pm 0.04 t$-types. Meanwhile, the mean absolute deviation is $1.05 \pm 0.03 t$-types.

\footnotetext{
15 One of these $3 \mathrm{SNe}, \mathrm{SN} 2005 \mathrm{md}$, reported by Li et al. (2005) and initially classified by Modjaz et al. (2005) as a probable young type IIb SN, was shown to be in fact a new Galactic cataclysmic variable (Leonard 2010)
}

Table 3. Relation between the RC3 morphological types and $t$ values with ours.

\begin{tabular}{lcllc}
\hline \hline \multicolumn{2}{c}{ Ours } & & \multicolumn{2}{c}{ RC3 } \\
\cline { 1 - 2 } \cline { 5 - 5 } Type & $t$ & & Type & $t$ \\
\cline { 1 - 2 } $\mathrm{E}$ & -3 & & $\mathrm{cE}$ & -6 \\
$\mathrm{E}$ & -3 & & $\mathrm{E}$ & -5 \\
$\mathrm{E} / \mathrm{S} 0$ & -2 & & $\mathrm{E}^{+}$ & -4 \\
$\mathrm{~S} 0$ & -1 & & $\mathrm{~S}^{-}$ & -3 \\
$\mathrm{~S} 0$ & -1 & & $\mathrm{~S}^{0}$ & -2 \\
$\mathrm{~S} 0$ & -1 & & $\mathrm{~S} 0^{+}$ & -1 \\
$\mathrm{~S} 0 / \mathrm{a}$ & 0 & & $\mathrm{~S} 0 / \mathrm{a}$ & 0 \\
$\mathrm{Sa}$ & 1 & & $\mathrm{Sa}$ & 1 \\
$\mathrm{Sab}$ & 2 & & $\mathrm{Sab}$ & 2 \\
$\mathrm{Sb}$ & 3 & & $\mathrm{Sb}$ & 3 \\
$\mathrm{Sbc}$ & 4 & & $\mathrm{Sbc}$ & 4 \\
$\mathrm{Sc}$ & 5 & & $\mathrm{Sc}$ & 5 \\
$\mathrm{Scd}$ & 6 & & $\mathrm{Scd}$ & 6 \\
$\mathrm{Sd}$ & 7 & & $\mathrm{Sd}$ & 7 \\
$\mathrm{Sdm}$ & 8 & & $\mathrm{Sdm}$ & 8 \\
$\mathrm{Sm}$ & 9 & & $\mathrm{Sm}$ & 9 \\
$\mathrm{Im}$ & 10 & & $\mathrm{Im}$ & 10 \\
\hline
\end{tabular}
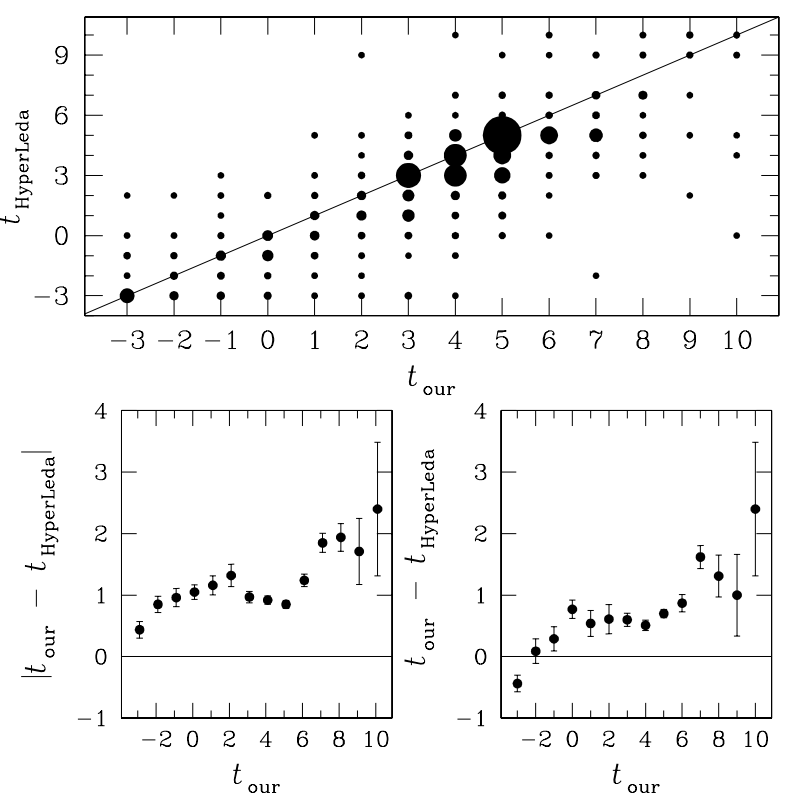

Fig. 6. Top: comparison of HyperLeda (reordered) versus our $t$ morphological types for 1313 host galaxies with available classifications. Point sizes are keyed to the number of objects. Bottom: the distributions of differences of our and HyperLeda $t$-types according to our classification. The error bars for the mean values in each bin are presented. The solid lines in each figure are added to visually better illustrate the deviation in classifications.

Table 4 shows the results of a linear regression between the HyperLeda types and ours. The relation between the two measures of $t$ has a slope significantly different than unity, with a best fit value of 0.93 , and the residuals from this trend have a dispersion close to 1.5 types.

A similar trend was already found by Nair \& Abraham (2010), who recently released a morphological catalog of 14034 visually classified galaxies $(0.01<z<0.1)$.

We found that approximately $10 \%$ (125 hosts) of the 1313 galaxies in common with HyperLeda have $t$-types that are dramatically different $\left(\left|t_{\text {our }}-t_{\text {HyperLeda }}\right| \geq 3\right)$. In Fig. 7, we present several extreme cases where the difference between 
A\&A 544, A81 (2012)
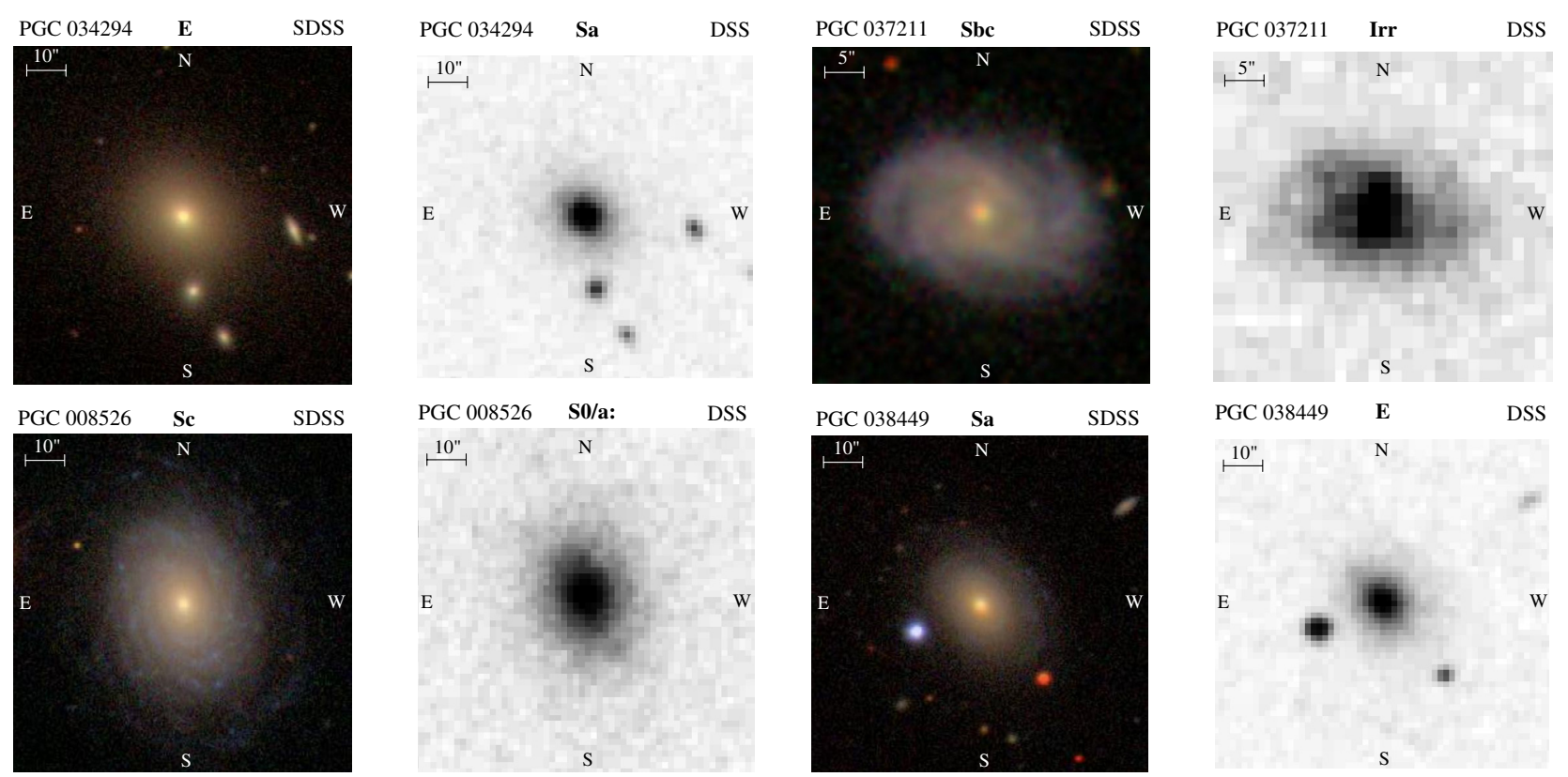

Fig. 7. Examples of the SDSS DR8 and DSS I images of SN host galaxies representing the cases when $\left|t_{\text {our }}-t_{\text {HyperLedal }}\right| \geq 3$. The PGC object identifier is listed at the top with our (left) and the HyperLeda (mainly selected from the RC3, right) classifications. In all images, north is up and east to the left.

Table 4. Comparisons of HyperLeda and SDSS DR7 measurements with ours for SN host galaxies.

\begin{tabular}{lcccc}
\hline \hline Quantity & Ref. cat. & $a$ & $b$ & Dispersion \\
\hline$t$ & HyperLeda & $0.93 \pm 0.01$ & $-0.29 \pm 0.05$ & 1.48 \\
$\log a$ & HyperLeda & $0.93 \pm 0.00$ & $0.06 \pm 0.01$ & 0.06 \\
$\log a$ & SDSS & $0.87 \pm 0.01$ & $0.25 \pm 0.01$ & 0.07 \\
$b / a$ & HyperLeda & $0.94 \pm 0.01$ & $0.04 \pm 0.01$ & 0.08 \\
$b / a$ & SDSS & $0.97 \pm 0.01$ & $0.01 \pm 0.01$ & 0.07 \\
PA & HyperLeda & $1.00 \pm 0.00$ & $-0.94 \pm 0.49$ & 5.72 \\
PA & SDSS & $1.00 \pm 0.00$ & $-0.56 \pm 0.47$ & 4.57 \\
mag & HyperLeda & $0.98 \pm 0.00$ & $0.66 \pm 0.06$ & 0.22 \\
mag & SDSS & $0.87 \pm 0.00$ & $2.01 \pm 0.06$ & 0.25 \\
\hline
\end{tabular}

Notes. Columns $3(a)$ and $4(b)$ represent the robust linear fits (with iterative rejection of outliers) of $x_{\text {theirs }}=a x_{\text {ours }}+b$. The last column (Dispersion) is computed as 1.483 times the median absolute deviation (which corresponds to $\sigma$ for Gaussian distributions) of the residuals from our best-fit trend. Cases with no measurements (given arbitrary values such as 99) have been discarded. The SDSS magnitude is cModelMag in the $g$-band. The PA dispersion is in degrees.

morphological type codes is $\geq 3$. Color images are taken from the SDSS DR8 on which our classification was performed, while the grayscale images are from the photographic plates given in the Digitized Sky Survey I (DSS I), as the RC3 classification is mostly based on these or similar plates. In many cases, photographic plates suffer from the narrow dynamical range that causes saturation as well as underexposure, and also from their non-linear response functions (e.g., Naim et al. 1995). A detailed comparative study of the SDSS and DSS images of hosts, when $\left|t_{\text {our }}-t_{\text {HyperLeda }}\right| \geq 3$, allows us to emphasize that in nearly all cases, the overexposure as well as low resolution of the photographic plates cause late-type galaxies of high surface brightness to be misclassified as early-type in the RC3. We have found a handful of cases with the opposite trend: E/S0 misclassified as spirals in RC3, mainly due to the heterogeneous nature of morphological data sets in the HyperLeda.

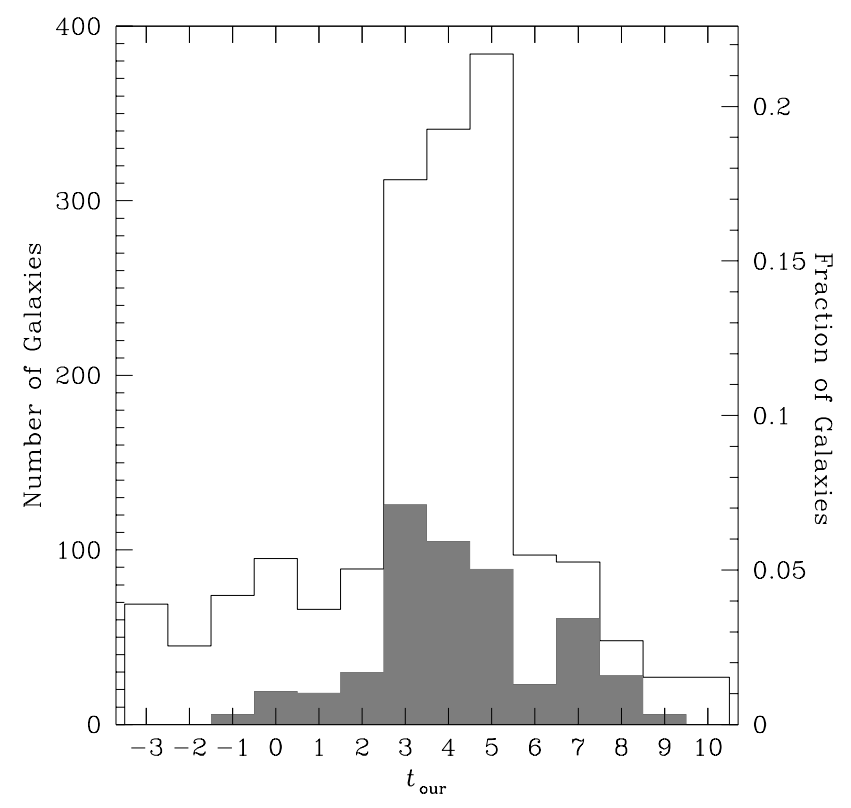

Fig. 8. Distributions of morphological types and presence of bars (shaded region) in 1767 classified hosts.

Baillard et al. (2011) have recently released the EFIGI (Extraction de Formes Idéalisées de Galaxies en Imagerie) catalogue; a multi-wavelength database specifically designed to densely sample all Hubble types. Their imaging data were obtained from the SDSS DR4 for a sample of 4458 PGC galaxies. This catalog includes 453 galaxies from our sample. We found very good agreement between the EFIGI morphological classifications and ours.

Figure 8 shows the distribution (see Table 2) of SN host galaxies with respect to our $t$-type. This histogram shows that the intermediate types $\mathrm{Sb}, \mathrm{Sbc}$, and $\mathrm{Sc}$ are the most frequent. Only Sm and Im types, which are intrinsically faint, have fewer than 30 galaxies per type. 
A. A. Hakobyan et al.: Supernovae and their host galaxies. I.

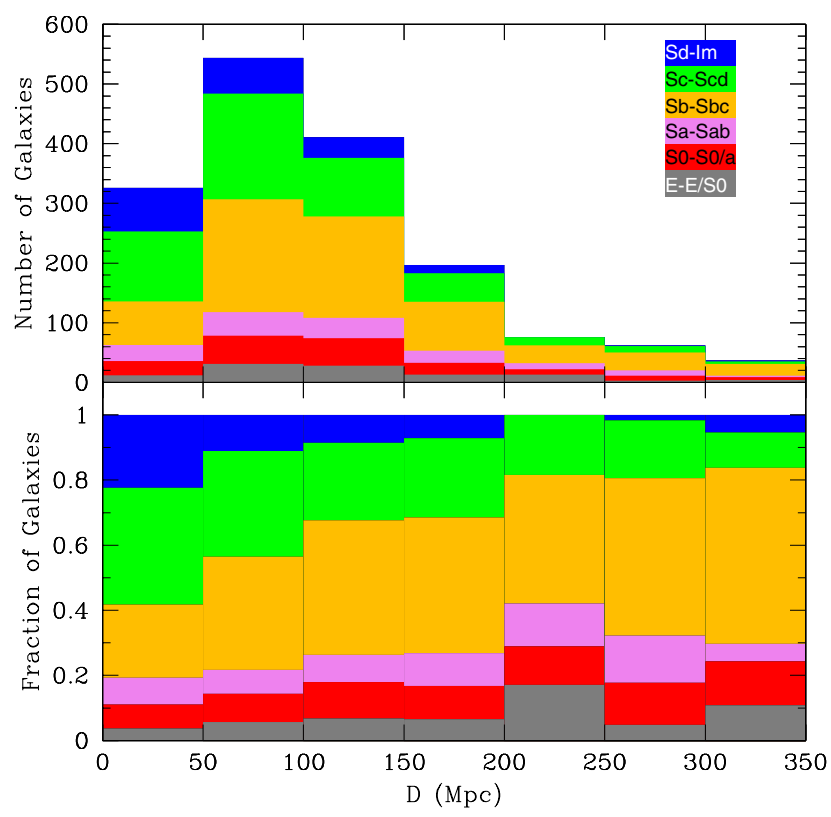

Fig. 9. Top: distribution of morphological types as a function of distance. The types of SN host galaxies have been grouped into the following broad classes: E and E/S0 (gray), S0 and S0/a (red), Sa and Sab (magenta), Sb and Sbc (orange), Sc and Scd (green), and hosts Sd to Im (blue). Bottom: fractional distribution of morphological types (with the same morphological groups) as a function of distance.

In the top panel of Fig. 9 we present the histogram of morphological types as a function of distance. The types of host galaxies have been grouped into the following broad classes: $\mathrm{E}$ and E/S0 (gray), S0 and S0/a (red), Sa and Sab (magenta), Sb and Sbc (orange), Sc and Scd (green), and hosts Sd to Im (blue). The bottom panel of Fig. 9 shows the fractional distribution of types of the same morphological groups as a function of distance. Host galaxies with $D<200 \mathrm{Mpc}$ include nearly the whole range in morphological types. At the same time, the late-type hosts are preferentially distributed in the lower distance bins, while early-types are more populated at the higher distances. At distances $D<200 \mathrm{Mpc}$, Sd to Im type hosts represent $12 \%$ of the classified galaxies.

At large distances, spiral galaxies can be under-represented, because spiral arms are difficult to resolve (see, e.g., Nair \& Abraham 2010; Baillard et al. 2011). Hence, the most massive/luminous early-type galaxies prefer the higher distances, while the least massive/luminous late-type galaxies are more populated at the lower distances. This is also the selection effect on the SN type: bright SNe Ia, exploding also in E galaxies, can be found more easily than fainter CC SNe, exploding only in late-type galaxies (e.g., Leaman et al. 2011). Therefore, the sample of CC SNe hosts is closer on average than the sample of SNe Ia host galaxies.

\subsection{Comparison of presence of bars in host galaxies}

A proper detection of barred structures of hosts is very important when constraining the nature of the SNe progenitors by comparing their distribution within host galaxies with the distributions of stellar populations and ionized gas in the disks (e.g., Petrosian et al. 2005; Hakobyan et al. 2009). Below, we have carried out a comparative study to find differences in the detection of barred structures of host galaxies between our classified sample and HyperLeda.

Figure 8 presents the distribution (see Table 2) of hosts with or without bars as a function of $t$-type. Roughly $29 \%$ of our 1767 classified galaxies have bars. The barred fraction is highest in types $\mathrm{Sb}$, Sbc, and Sc. A detailed comparison with HyperLeda reveals that in 378 galaxies among our 1767 in common $(21 \%)$, HyperLeda fails to detect the bar that we visually detect on the SDSS images or conversely detects a bar when we don't. In Fig. 10, we present examples of hosts galaxies with discrepancies in bar detection between HyperLeda and us. Given their superior angular resolution and 3-color representations, the SDSS images offer a much more reliable source for bar detection than do the plate-based images on which most of the HyperLeda classifications were performed.

Inspecting these cases of discrepancies in bar detection, we conclude that HyperLeda fails to show bars in both high central surface brightness early-type galaxies and late-type galaxies with low surface brightness bars. We found that bars tend to be incorrectly detected in HyperLeda galaxies of high inclination $\left(i>70^{\circ}\right)$. The remaining cases of detection discrepancies are again due to the heterogeneous nature of the HyperLeda data sets. Note that we may also have missed weak bars because of inclination effects, or that in some cases the SDSS images of hosts may be too shallow to detect bars. For instance, among our S0-Sm galaxies with inclinations $i<70^{\circ}$, the average bar fraction is $(37 \pm 1) \%$ whereas for hosts with inclinations $\geq 70^{\circ}$ the average bar fraction is only $(11 \pm 2) \%$.

\subsection{Comparison of isophotal measurements of host galaxies}

We checked the differences of our measurements of $g$-band major axes with the HyperLeda $B$-band diameters $\left(D_{25}\right)$ as well as with the SDSS $g$-band isophotal major axes (isoA). Table 4 shows the results of a linear regression between the HyperLeda, SDSS isophotal measurements and ours.

In the top left panels of Figs. 11 and 12 we show the discrepancy of the major diameters of the host galaxies between the samples. Our measured $g$-band diameters are systematically larger than the HyperLeda $D_{25}$ diameters in $B$-band for all the morphological types of hosts. Our diameters are greater than those in the HyperLeda on average by a factor of $1.32 \pm 0.01$. This level of discrepancy is not unexpected, given that our sizes are measured at the $\mu_{g}=25 \mathrm{mag} \operatorname{arcsec}^{-2}$ isophotal level. With the transformation equation, $B=g+0.39(g-r)+0.21$ (Jester et al. 2005, for all stars with $R-I<1.15$ ), and given our mean $g-r \simeq 0.7$ color, our $g$-band measurements are performed at the equivalent of the $\left\langle\mu_{B}\right\rangle \simeq 25.48$ isophote, hence our greater host galaxy sizes.

For the data comparisons with SDSS, we used DR7 instead of DR8, because DR7 includes isophotal photometric quantities. Before comparing the samples of classified E-Im galaxies we excluded 96 objects with unreliable SDSS measurements that differ from our measurements by more than a factor of 2 . We considered the data for these galaxies as incorrectly measured in SDSS. Our diameters are in good agreement with isoA, and greater only on average by a factor of $1.01 \pm 0.01$. In general, the SDSS measurements are unreliable for objects larger than $\sim 100$ arcsec (e.g., Baillard et al. 2011). Still, for objects smaller than $100^{\prime \prime}$, we find scatters corresponding to factors of $33 \%$ (with HyperLeda) and 16\% (with SDSS DR7).

In the top right panels of Figs. 11 and 12 we present the differences of our measured axial ratios and that of the HyperLeda as well as isoB/isoA of the SDSS DR7. The majority ( 94\%) 
A\&A 544, A81 (2012)
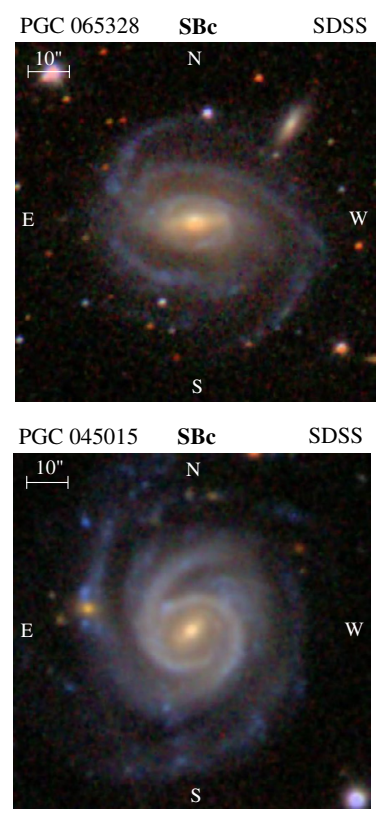
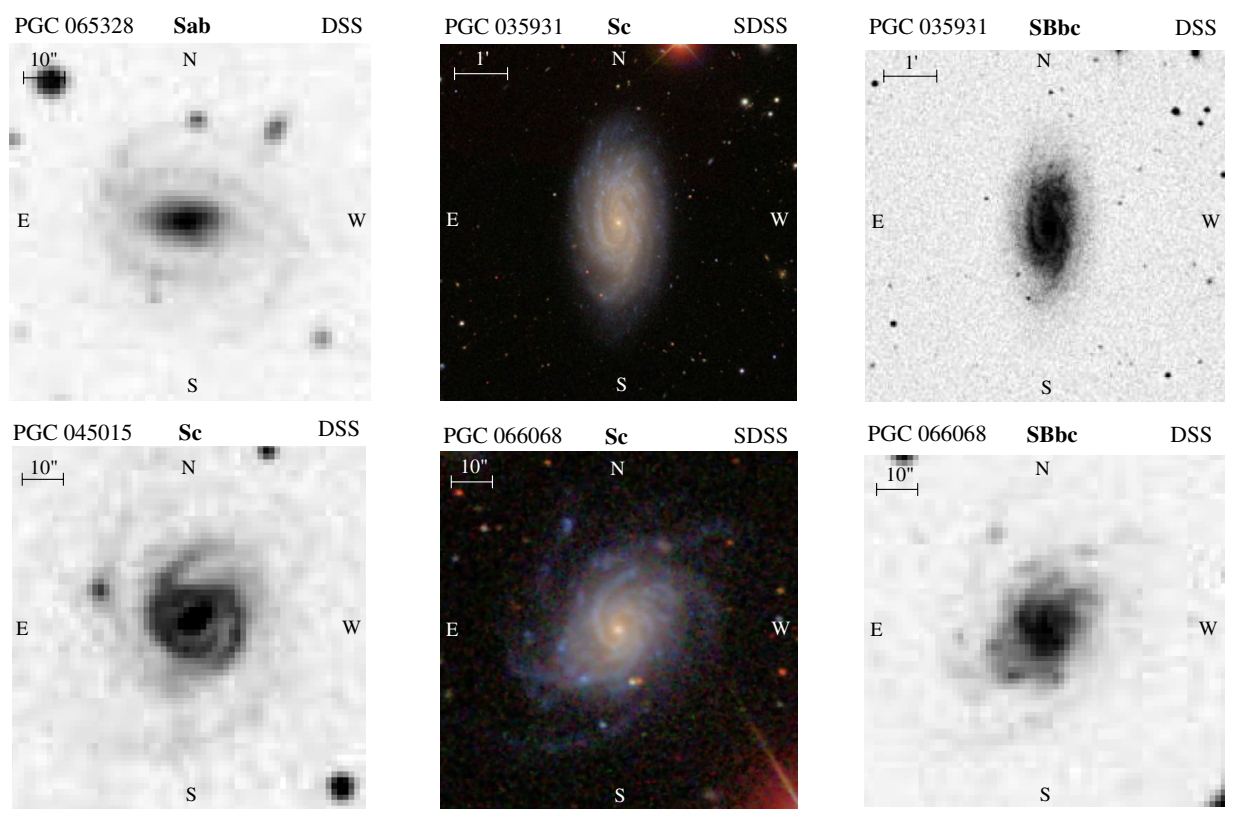

Fig. 10. Examples of SDSS DR8 and DSS I images of SN host galaxies representing the cases of discrepancies in bar detection between HyperLeda and us. The PGC object identifier is listed at the top with our (left) and the HyperLeda (mainly selected from the RC3, right) classifications. In the two galaxies on the left, we detect bars, while HyperLeda does not, while in the two galaxies on the right, HyperLeda claims that there is a bar while we dot not see any on our higher-resolution 3-band images. In all images, north is up and east to the left.

of E-Im galaxies, for which the axial ratios are available in our and other measurements, these axial ratios are consistent within 0.2. The mean deviation of our measurements from that of the HyperLeda is $0.005 \pm 0.003$, and from isoB/isoA is $0.015 \pm 0.003$. The MADs are 0.055 with HyperLeda and 0.052 with SDSS DR7. In fact, after correction for trends, the residuals show a robust dispersion of $\sim 0.1$. There is no dependence of residuals on the morphological types of host galaxies.

The position angles (PAs) of the major axes were determined at the same $\left(\mu_{g}=25 \mathrm{mag} \operatorname{arcsec}^{-2}\right)$ isophotal level as the measurements of angular diameters. Comparisons of our PA measurements with the HyperLeda and SDSS DR7 (isoPhi) determinations are shown in the bottom left panels of Figs. 11 and 12.

There are non-negligible fractions of cases where $\mathrm{PA}_{\text {our }}+$ $\mathrm{PA}_{\text {theirs }}=180^{\circ}$, especially for $\mathrm{PA}_{\text {our }} \simeq 0^{\circ}$ or $180^{\circ}$, for which small errors can (for the correct sign) flip the PA to $180^{\circ} \mathrm{mi}-$ nus its true value. To avoid these unfair extreme outliers in our comparisons, we redistributed the values of differences of PAs such that we considered the $\triangle \mathrm{PA}-180^{\circ}$ or $\triangle \mathrm{PA}+180^{\circ}$ when the differences were $>90^{\circ}$ or $<-90^{\circ}$, respectively.

With these corrections, the mean difference between HyperLeda's PAs and ours is $0.9 \pm 0.5$, while the MAD is 4.9 . The comparison with the PAs from SDSS DR7 (isoPhi) yields a mean deviation of $0.5 \pm 0.6$ and a MAD of 4.3 . In both cases, $85 \%$ of the host galaxies have PAs consistent within $20^{\circ}$ with those of HyperLeda or SDSS. The scatter in the bottom left panel of Fig. 11 may be due to the fact that the HyperLeda values correspond to measurements made at $\mu_{B}=25 \mathrm{mag} \mathrm{arcsec}{ }^{-2}$, whereas ours are made at typically lower surface brightness thresholds: $\left\langle\mu_{B}\right\rangle=\sim 25.48 \mathrm{mag} \operatorname{arcsec}^{-2}$. Inspections of the remaining cases with large discrepancies show that they are mostly contributed by peculiar and low surface brightness galaxies or objects in interacting/merging systems. In addition, the PA is hard to determine when the galaxy is face-on, because for any given elliptical aperture it is ill-defined; $97 \%$ of galaxies with $b / a \leq 0.5$ are consistent within $20^{\circ}$ of $\mathrm{PA}$, whereas only $80 \%$ of hosts with $b / a>0.5$ have the same consistency. Again, there is no dependence of discrepancies on the morphological types of galaxies. For more detailed inspection and explanation of this effect see Petrosian et al. (2007).

We also compared our $g$-band magnitude measurements to the HyperLeda $B$-band and to the SDSS DR7 Composite Model Magnitude (cModelMag) determinations in the $g$-band, which are measured from the linear combination of the exponential and de Vaucouleurs profiles that fit best the $g$-band SDSS images. Also, there is excellent agreement between cModelMag and Petrosian (1976) magnitudes of galaxies. Although, the cModelMag and Petrosian magnitudes are not identical, there is an offset of $0.05-0.1 \mathrm{mag}$ but this is within errors of our elliptical aperture measurements. The results of magnitude comparisons are presented in the bottom right panels of Figs. 11 and 12. The relation between the HyperLeda magnitudes and ours is quite linear with a slope of 0.98 (our magnitudes are slightly brighter relative to theirs at the bright end). The residuals from our robust linear fit between the two magnitudes is $0.22 \mathrm{mag}$.

The mean difference between our magnitudes and those of HyperLeda is $-0.42 \pm 0.01$, while the mean absolute difference is $0.47 \pm 0.01$. It is clear that most of the measurements agree well with each other once the magnitudes are brought into the same system. For $\sim 65 \%$ of galaxies our and HyperLeda magnitude differences are less than $0.5 \mathrm{mag}$, which corresponds to $0.02 \mathrm{mag}$ after converting the $g$-band into $B$-band. Only for $\sim 7 \%$ of the hosts galaxies the magnitude difference is equal or larger than $1 \mathrm{mag}$, hence $0.52 \mathrm{mag}$ after the conversion. These galaxies are mostly of types Sc to Im.

The mean difference between our magnitudes and the SDSS cModelMag photometry is $-0.21 \pm 0.01$. The MAD is 0.19 mag. There is a trend where we measure brighter magnitudes than SDSS for the brightest galaxies. Despite some curvature in the relation between the two magnitude estimates, we fit a lines, and find $m_{\text {SDSS }}=0.87 m_{\text {ours }}$, with a dispersion of $0.25 \mathrm{mag}$ about this relation. 
A. A. Hakobyan et al.: Supernovae and their host galaxies. I.
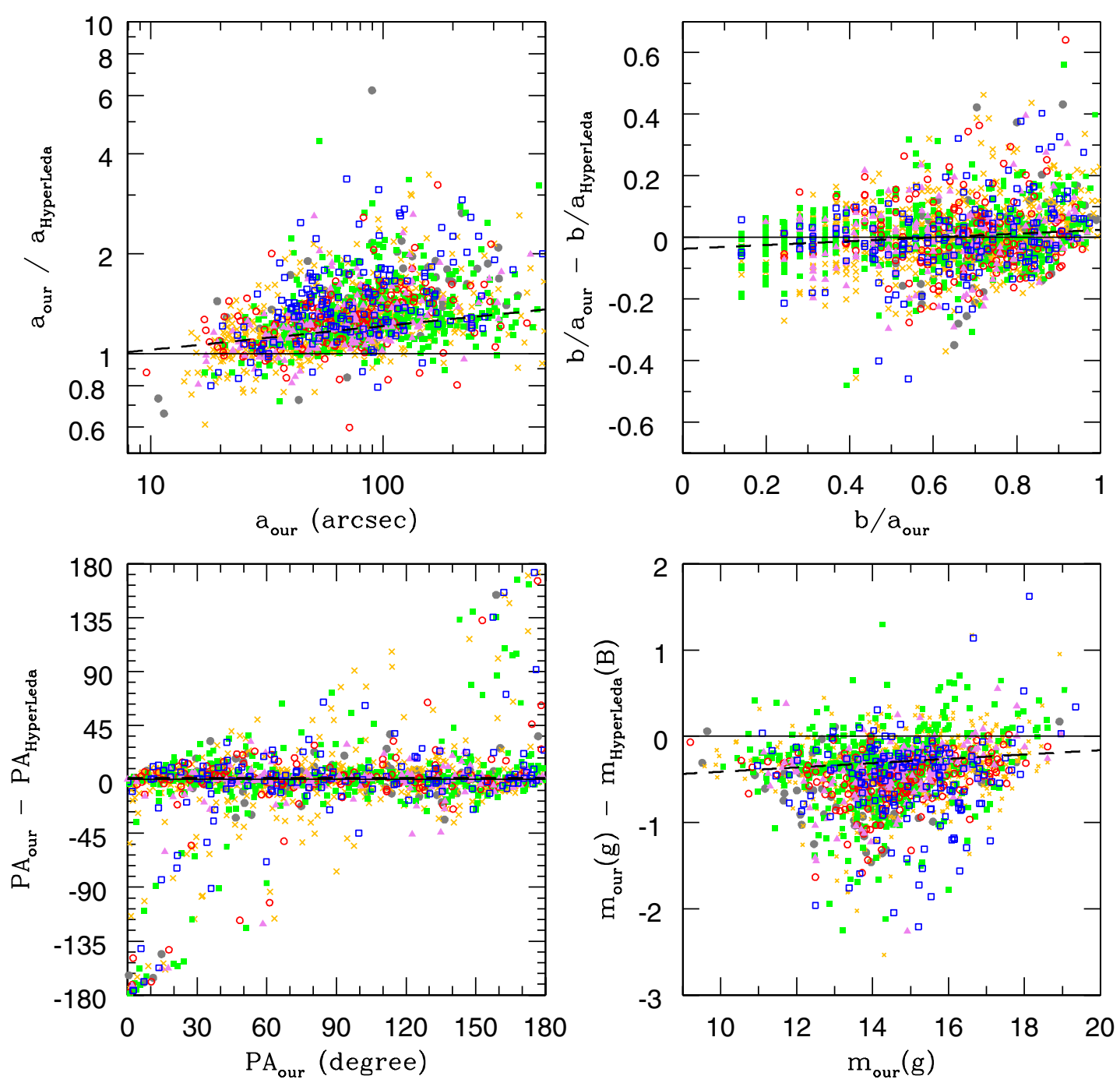

Fig. 11. Top left: comparison between our measurements of major axes of E-Im galaxies and those of the HyperLeda in the B-band. Top right: comparison between measured axial ratios and those of the HyperLeda. Bottom left: comparison between our measurements of position angles and those of the HyperLeda. Bottom right: comparison between our measurements of apparent $g$-band magnitudes and that in the $B$-band of the HyperLeda. The color coding corresponds to Fig. 9: E-E/S0 (gray filled circles), S0-S0/a (red open circles), Sa-Sab (magenta triangles), Sb-Sbc (orange crosses), Sc-Scd (green filled squares), and Sd-Im (blue open squares). The solid lines in each figure are added to visually better illustrate the deviations. The dashed lines are best fit linear trends from Table 4.

For $\sim 81 \%$ of galaxies our and the SDSS magnitude differences are less than $0.5 \mathrm{mag}$. For $\sim 5 \%$ of the objects the magnitude differences are equal to or larger than $1 \mathrm{mag}$. For faint galaxies, the SDSS measurements algorithm overestimates the cModelMag fluxes, while for bright galaxies it underestimates the fluxes. This trend is stronger for late-type galaxies. Again, we explain the presence of the large scatters, especially for the bright galaxies, by the unreliable SDSS photometric measurements for objects larger than $\sim 100$ arcsec (e.g., Baillard et al. 2011). We also performed the same analysis using the cModelMag of SDSS DR8 instead of DR7, and found the same behavior for the photometric bias.

In addition, we checked the influence of nuclear activity on the discrepancies of the photometric measurements. Indeed, since the SDSS total magnitudes for extended objects (ModelMag and cModelMag) are based on single-component fits, we could expect that such fitting will perform poorly for AGNs with relatively bright nuclei. The result is negative: there is no dependence of the discrepancies on the nuclear activity of the host galaxies.
The top left panel of Fig. 13 shows the distribution of inclination angles for morphologically classified disk (S0-Sm) galaxies. There is a clear deficit of SN host galaxies having small and large inclinations. We thus share the view with Leaman et al. (2011), who found a deficit of LOSS galaxies with small inclinations and explained this deficit by limits of the precision on the major and minor axes. Indeed, it is very difficult to measure inclinations smaller than $20^{\circ}$ from elliptical aperture measurements applied to nearly face-on galaxies. The lack of galaxies with large inclinations can be explained by a bias in the discovery of SNe (see also the middle panel of Fig. 14) in highly inclined spirals (e.g., Cappellaro \& Turatto 1988; van den Bergh \& Tammann 1991; Cappellaro et al. 1997).

The top right panel of Fig. 13 presents the distribution of corrected $g$-band absolute magnitudes for the classified galaxies. In comparison with the distribution of SDSS galaxies (dashed histogram), the $\mathrm{SN}$ host galaxies are more luminous. A Kolmogorov-Smirnov (KS) test indicates that the more luminous distribution of SN host galaxy magnitudes, relative to the SDSS galaxies in general, cannot be obtained by chance with 



Fig. 12. Top left: comparison between our measurements of major axes of E-Im galaxies and isophotal major axes in the $g$-band of the SDSS DR7. Top right: comparison between measured axial ratios and those of the SDSS DR7. Bottom left: comparison between our measurements of position angles and those of the SDSS DR7. Bottom right: comparison between our measurements of apparent $g$-band magnitudes and Composite Model Magnitudes of the same band in the SDSS DR7. The color and symbol coding corresponds to Fig. 11. The solid lines in each figure are added to visually better illustrate the deviations. The dashed lines are best fit linear trends from Table 4.

more than $0.1 \%$ probability. This distribution again suffers from a selection effect on $\mathrm{SN}$ productivity, since the rate of $\mathrm{SNe}$ depends on the luminosity or stellar content of the host galaxies (e.g., van den Bergh \& Tammann 1991; Cappellaro et al. 1997; Li et al. 2011; Hakobyan et al. 2011). Therefore, our sample of classified host galaxies is biased toward bright galaxies. For comparison, the distribution of $g$-band absolute magnitudes of the SDSS Main Galaxy sample is also shown.

The bottom panel of Fig. 13 shows the $g$-band luminosity of the same galaxies as a function of their distance. Galaxy luminosities were derived from absolute magnitudes, assuming that $g$-band absolute magnitude of the Sun is 5.45 (Blanton et al. 2003). The luminosities of late-type (Sd-Im) hosts are on average 5 times lower than those of early-type (E-E/S0) galaxies. This trend is clearly seen in the bottom panel of Fig. 13. The average $g$-band luminosities in different distance bins are also plotted. At greater distances, the low-luminosity host galaxies are lost due to flux limitations. Thus, Malmquist bias causes the average $g$-band luminosity to increase with increasing distance. The databases become progressively incomplete for low-luminosity galaxies at greater distances. This was already mentioned by Leaman et al. (2011) for the LOSS galaxy sample.

\subsection{Distribution of SN types}

In Table 5, we present the distribution of SN types according to the morphology of their host galaxies in an analogous fashion as in Table 5 of Barbon et al. (1999). It is clear that most SNe are found in spiral hosts. The percentage of unclassified $\mathrm{SNe}$ is $\sim 18 \%$ of the total sample. Approximately half of the unclassified SNe were discovered before 2000.

The left panel of Fig. 14 shows the number distribution of $\mathrm{SN}$ types as a function of host morphology. There is a significant difference between the distribution of the SN Ia hosts and that of CC SN hosts, while types Ibc and II SN hosts have similar distributions. These trends were previously reported by van den Bergh et al. $(2002,2003,2005)$ and Leaman et al. (2011).

It is generally believed that hosts of CC SNe are objects with young stellar populations (generally spiral or irregular galaxies), 
A. A. Hakobyan et al.: Supernovae and their host galaxies. I.

Table 5. Distribution of SN types according to the morphological classification of the host galaxies.

\begin{tabular}{lccccccccccccccccc}
\hline \hline & $\mathrm{E}$ & $\mathrm{E} / \mathrm{S} 0$ & $\mathrm{~S} 0$ & $\mathrm{~S} 0 / \mathrm{a}$ & $\mathrm{Sa}$ & $\mathrm{Sab}$ & $\mathrm{Sb}$ & $\mathrm{Sbc}$ & $\mathrm{Sc}$ & $\mathrm{Scd}$ & $\mathrm{Sd}$ & $\mathrm{Sdm}$ & $\mathrm{Sm}$ & $\mathrm{Im}$ & $\mathrm{S}$ & Unclassified & All \\
\hline I & 4 & 2 & 3 & 5 & 4 & 4 & 8 & 6 & 8 & 1 & 1 & 1 & 3 & 0 & 4 & 18 & 72 \\
$\mathrm{Ia}$ & 61 & 35 & 51 & 73 & 31 & 50 & 122 & 125 & 114 & 28 & 22 & 9 & 2 & 7 & 199 & 1061 & 1990 \\
$\mathrm{Ib}$ & 1 & 0 & 1 & 0 & 1 & 2 & 6 & 9 & 14 & 5 & 4 & 3 & 2 & 1 & 5 & 9 & 63 \\
$\mathrm{Ib} / \mathrm{c}$ & 0 & 0 & 0 & 1 & 3 & 2 & 5 & 12 & 10 & 1 & 2 & 3 & 0 & 0 & 4 & 8 & 51 \\
$\mathrm{Ic}$ & 0 & 0 & 0 & 0 & 1 & 4 & 15 & 30 & 18 & 9 & 10 & 1 & 1 & 0 & 8 & 23 & 120 \\
II & 0 & 0 & 2 & 4 & 9 & 9 & 98 & 121 & 182 & 39 & 39 & 15 & 9 & 12 & 55 & 116 & 710 \\
$\mathrm{IIb}$ & 0 & 0 & 0 & 0 & 2 & 0 & 4 & 9 & 10 & 5 & 3 & 5 & 2 & 1 & 6 & 3 & 50 \\
IIn & 0 & 0 & 0 & 1 & 2 & 0 & 11 & 12 & 28 & 9 & 3 & 3 & 3 & 2 & 9 & 27 & 110 \\
Unclassified & 9 & 9 & 18 & 16 & 17 & 21 & 68 & 69 & 65 & 14 & 17 & 10 & 6 & 4 & 55 & 312 & 710 \\
\hline All & 75 & 46 & 75 & 100 & 70 & 92 & 337 & 393 & 449 & 111 & 101 & 50 & 28 & 27 & 345 & 1577 & 3876 \\
\hline
\end{tabular}

Notes. All SNe types include uncertain (““” or “?”) and peculiar (“pec”) classifications. Type II SNe include subtypes II P and II L. Types I, Ia, and II include also few SNe classified from the light curve only, these SNe are labeled by "*”" symbols in the total sample.
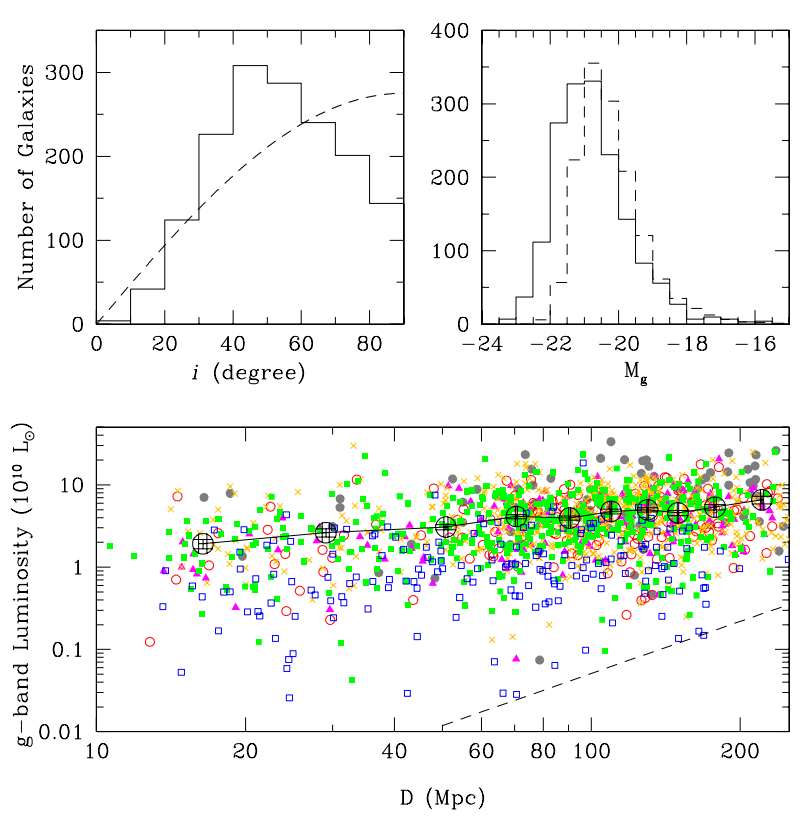

Fig. 13. Top left: distribution of the inclination angles for disk (S0-Sm) galaxies. The dashed curve represents the expected random distribution. Top right: distribution of corrected $g$-band absolute magnitudes for the classified (E-Im) galaxies. The dashed histogram shows the distribution of $g$-band absolute magnitudes of the SDSS Main Galaxy sample (the values are divided by 500 for the sake of clarity). Bottom: the $g$-band luminosity of the same galaxies as a function of their distance. The color and symbol coding corresponds to Fig. 11. The average luminosity in different distance bins is overplotted as big open circles with error bars of the mean values. The dashed line represents the selection limit of the SDSS Main Galaxy spectroscopic sample $(r \leq 17.77)$ for the extinction corrected Petrosian magnitude, assuming $g-r=0.64$.

while the old stellar population of early-type galaxies can produce only SNe Ia (e.g., van den Bergh \& Tammann 1991; Cappellaro et al. 1999). Nevertheless, among the morphologically classified host galaxies of $\mathrm{CC} \mathrm{SNe}$ in our sample, we have found 4 cases (2000ds [Ib] in PGC 025915, 2006ee [II] in PGC 007536, 2007ke [Ib] in PGC 010959, and 2009fe [II] in SBS 1646+499) in which the host has been classified as E or S0, in apparent contradiction to this conventional view. Figure 15 presents the cases of CC SNe in early-type hosts.

Hakobyan et al. (2008) already reported and investigated in detail two cases of such CC SNe in early-type galaxies (2000ds
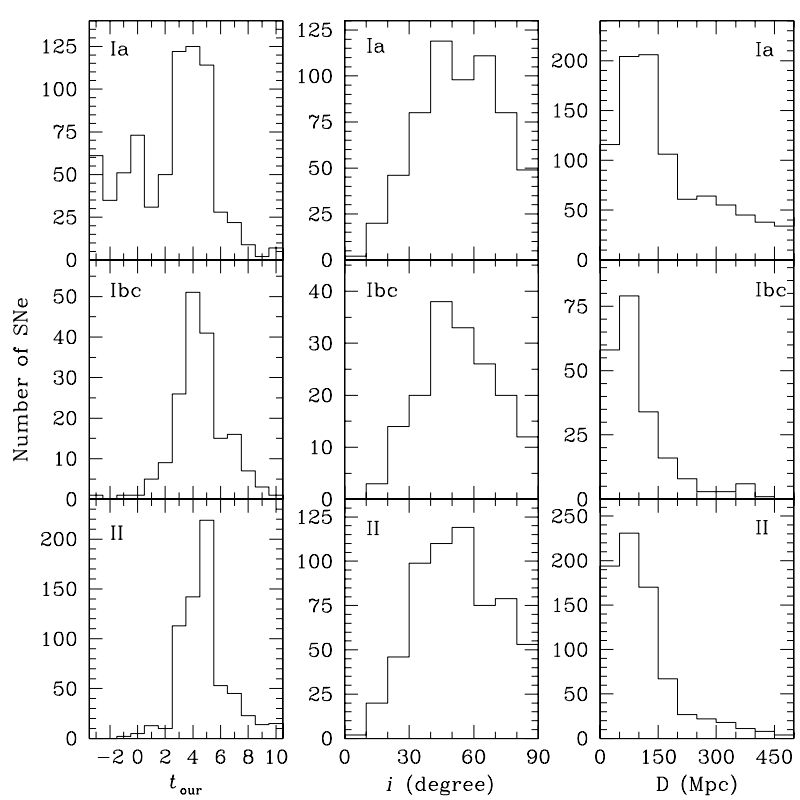

Fig. 14. Left: distribution of different types of $\mathrm{SNe}$ as a function of the host galaxy $t$-type. Middle: distribution of $\mathrm{SNe}$ as a function of the inclination of the spiral hosts. Right: distribution of different types of $\mathrm{SNe}$ as a function of the distance of their host galaxy.

and 2006ee). The host galaxy of SN 2000ds (Filippenko \& Chornock 2000) has been confirmed to be an S0, with a central region showing dust and a disky central gas distribution (e.g., Hakobyan et al. 2008). According to the outer isophotal structure and radial surface brightness profile of the host of SN 2006ee (Puckett et al. 2006), this must be an S0 galaxy. It has been shown that the surface brightness distribution has some small degree of asymmetry in the region to the south-southwest of the nucleus (Hakobyan et al. 2008). Here, we suspected the presence of an embryonic spiral arm. We classified the host galaxy of SN 2007ke (Filippenko et al. 2007) as type E, this classification is also given in both NED and HyperLeda. It is in interacting system and is a member of the cluster of galaxies. The host galaxy of SN 2009fe (Kasliwal et al. 2009) is classified as an uncertain type S0. The same morphological type is reported in Gyulzadyan et al. (2011). In NED, it is classified as a blazar (Seyfert 1). This object also shows $1.4 \mathrm{GHz}$ radio continuum emission (Condon et al. 1998). However, more detailed inspection on high resolution images is still required. In principle, the galaxy could have some diffuse spiral arms and be classified as S0/a but due to 

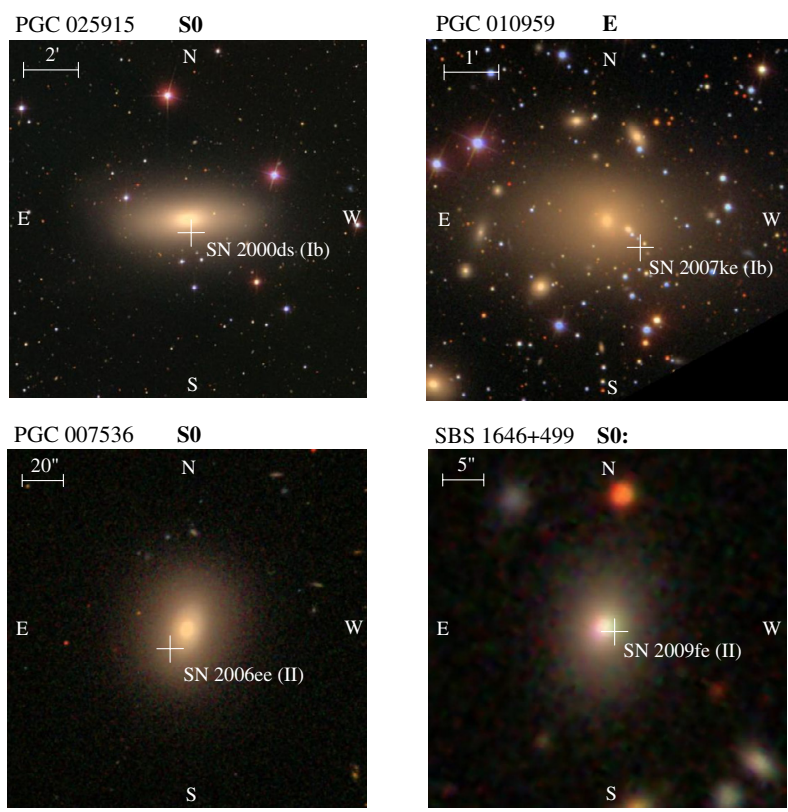

Fig. 15. SDSS images of early-type (E or S0) host galaxies with CC SNe. The objects identifiers are listed at the top with our morphological classification. The SN names, types, and positions (marked by cross sign) are also shown. In all images, north is up and east to the left.

insufficient resolution of the SDSS image at the distance of this object, it has been classified as an uncertain type SO.

The presence of $\mathrm{CC} \mathrm{SNe}$ in early-type galaxies can be interpreted as an additional indication that residual star formation episodes also take place in E or S0 galaxies, due to merging/accretion or interaction with close neighbors. Recently, Kaviraj et al. (2009) have found that the recent star formation is likely to be driven by minor mergers, which seems to fit with our interpretation as well. Meanwhile, using rest-frame UV photometry of early-type galaxies in the nearby Universe, Kaviraj et al. (2007) suggested that low-level recent star formation is widespread in nearby early-type galaxies. The situation is also very similar at intermediate redshifts (Kaviraj et al. 2008). Hence, the detection of SNe II in early-type galaxies is expected, but at lower frequency than type Ia.

The middle panel of Fig. 14 shows the number distribution of different types of SNe as a function of the inclination of S0-Sm hosts (see also the top left panel of Fig. 13 and its explanation). The KS test suggests that there is no significant difference between the distributions of types Ia and CC SNe. The same behavior occurs when comparing type Ia SNe separately with types Ib and II SNe. A similar trend was mentioned by Cappellaro et al. (1997).

The right panel of Fig. 14 shows the number distribution of $\mathrm{SN}$ types as a function of distance. All the major types of $\mathrm{SNe}$ are peaked at $50-100 \mathrm{Mpc}$. It is clear that the sample of $\mathrm{SNe}$ is largely incomplete beyond $\sim 100 \mathrm{Mpc}$. The distributions of types Ibc and II SNe are similar and display a sharp decline beyond $100 \mathrm{Mpc}$. Type Ia SNe, because of their comparatively high luminosity and the presence of dedicated surveys (e.g., SDSS SN Survey, ESSENCE etc.), are discovered at much greater distances than CC SNe. A similar behavior was also found by Leaman et al. (2011).

Table 6 displays the numbers of different types of SNe in hosts with different levels of nuclear activity. It is important to note that nuclear activity is affected by aperture bias
Table 6. Distribution of SN types according to the level of nuclear activity of the host galaxy.

\begin{tabular}{ccccccc}
\hline \hline Diagram & Activity & Ia & Ibc & II & Unclassified & All \\
\hline \multirow{5}{*}{ BPT } & Sy & 20 & 3 & 14 & 4 & 41 \\
& LINER & 77 & 8 & 41 & 40 & 166 \\
& C & 78 & 12 & 64 & 27 & 181 \\
& SF & 110 & 49 & 157 & 96 & 412 \\
\cline { 2 - 7 } & All & 285 & 72 & 276 & 167 & 800 \\
\hline \multirow{5}{*}{ WHAN } & Sy & 83 & 7 & 43 & 35 & 168 \\
& LINER & 38 & 7 & 24 & 19 & 88 \\
& SF & 199 & 66 & 218 & 121 & 604 \\
& RP & 235 & 13 & 72 & 86 & 406 \\
\cline { 2 - 7 } & All & 555 & 93 & 357 & 261 & 1266 \\
\hline
\end{tabular}

Notes. Each of the Seyfert (Sy) rows additionally include 6 BL AGN hosts. The RP row includes $77 \mathrm{P}$ galaxies. The column of unclassified $\mathrm{SNe}$ includes also type I SNe.

(e.g., Kewley et al. 2005). The SDSS spectra are taken with a fixed fiber size $\left(3^{\prime \prime}\right)$. For a nearby galaxy, the SDSS fiber covers the central nuclear region or its part, while for more distant case it covers a larger fraction of the galaxy (e.g., $120 \mathrm{pc}$ at $z=0.004$ but $2.7 \mathrm{kpc}$ at $z=0.1$ ). Hence, the activity data can be contaminated by the emission of circumnuclear regions or from the whole galaxy. The effect also depends on galaxy size, as for dwarf galaxies the fiber will cover a larger fraction of the total emission.

\section{Summary and perspectives}

In a series of papers, we will analyze how the different types of SNe events and their spatial distribution are correlated with the properties of the nuclei and global physical parameters of the host galaxies, as well as with the nearby and distant environments of these galaxies. In this first paper, we report the creation of large and well-defined database of $3876 \mathrm{SNe}$ and their 3679 host galaxies that are located on the SDSS DR8 coverage. This database has been created to increase the size of the sample relative to previous works, and to carry out morphological classification, as well as individual measurements of the global parameters of SN host galaxies to a more homogenous and detailed level. Throughout this work, we analyzed and discussed many selection effects that can bias future analyses.

We identified $91 \%$ of the host galaxy sample (3340 hosts with $3536 \mathrm{SNe}$ ) among which the SDSS identification of $\sim 1100$ hosts have been done for the first time. Using the SDSS multi-band images, photometric and spectral data, we provided accurate coordinates, heliocentric redshifts, morphological types, nuclear activity classes (RP, SF, C, LINER, and Sy), apparent $g$-band magnitudes, major axes $\left(D_{25}\right)$, axial ratios $(b / a)$, and position angles (PA) of the host galaxies.

During the mutual comparison of spectroscopic classification of SNe taken from the ASC and SSC and the literature search, we updated spectroscopic types for $67 \mathrm{SNe}$. We collected all the available data on 3166 SNe types when it was present in one of the SN catalogs or in the CBAT. Our total sample consists of $72 \mathrm{SNe}$ I, $1990 \mathrm{SNe}$ Ia, $234 \mathrm{SNe}$ Ibc, $870 \mathrm{SNe}$ II, and 710 unclassified SNe. In addition, the sample includes $3599 \mathrm{SNe}$ with offset data. We corrected offsets of $43 \mathrm{SNe}$ and calculated (relatively uncertain) coordinates for $332 \mathrm{SNe}$ with unavailable astrometry via offsets and accurate coordinates of the identified nuclei of their host galaxies. 
A. A. Hakobyan et al.: Supernovae and their host galaxies. I.

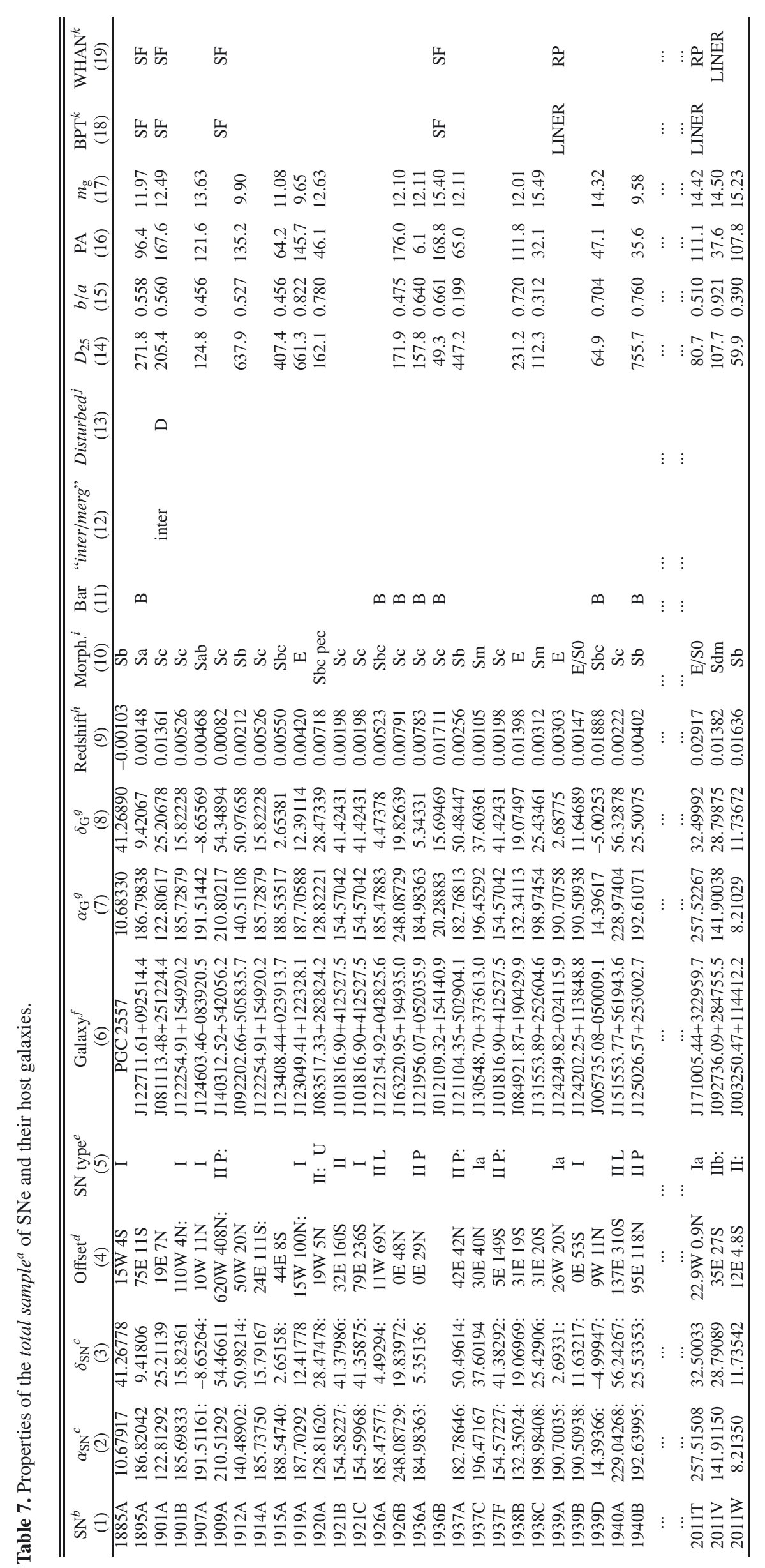

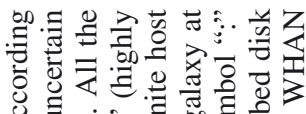

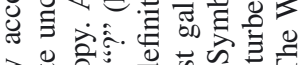

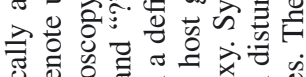

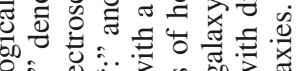
응.

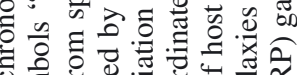

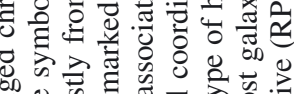

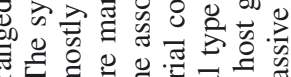

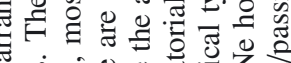
\&

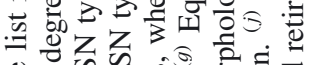

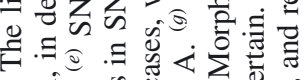

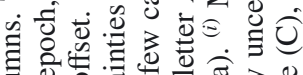

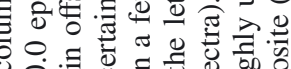

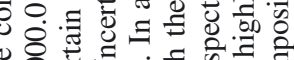

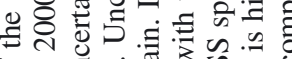

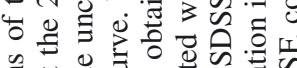

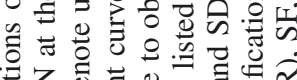

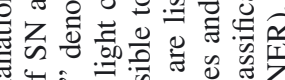
至 ช.

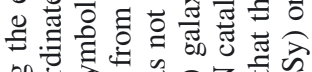

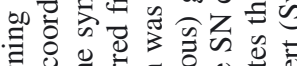

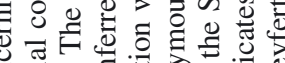

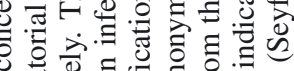

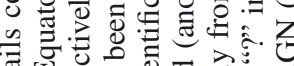
哥

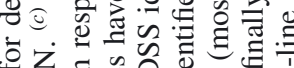

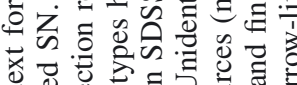
ख

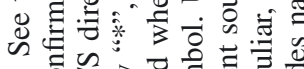

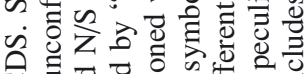

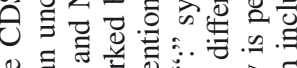

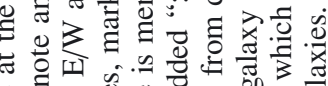

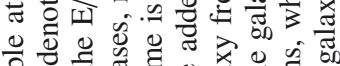
记

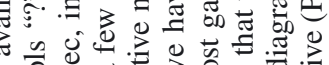
a 0 o 30 응 है

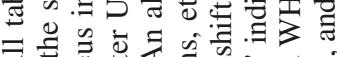

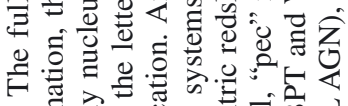

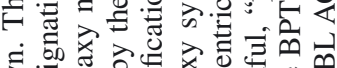

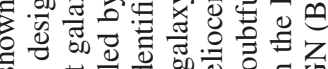

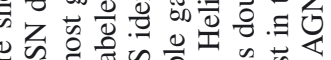

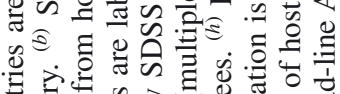

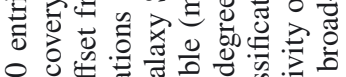
o d.

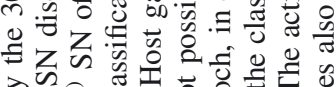

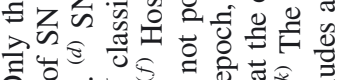

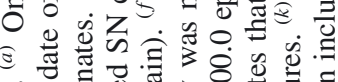

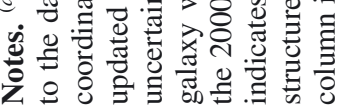


The morphological classification is available for 2104 host galaxies, which is $63 \%$ of our sample of identified galaxies and includes 73 hosts in interacting ("inter"), and 56 hosts in merging ("merg") systems. The total sample of host galaxies collects heliocentric redshifts for 3317 (90\%) galaxies. The $g$-band magnitudes, $D_{25}, b / a$, and PA are available for 2030 hosts of the morphologically classified sample of galaxies.

We also provided information on the nuclear activity of 1106 host galaxies by using the BPT and WHAN diagrams. The database also includes 6 broad-line AGNs and 77 passive $(\mathrm{P})$ galaxies without any emission features.

The creation of this large database will minimize possible selection effects and errors that often arise when data is collected from different sources and catalogs. In future papers of this series, we will largely use all photometric and spectral data to constrain the nature of SN progenitors, using their distribution within host galaxies of different morphological types and levels of nuclear activity.

Acknowledgements. A.A.H. and A.R.P. acknowledge the hospitality of the Institut d'Astrophysique de Paris (France) during their stay as visiting scientists supported by the Collaborative Bilateral Research Project of the State Committee of Science (SCS) of the Republic of Armenia and the French Centre National de la Recherche Scientifique (CNRS). V.Zh.A. and J.M.G. are supported by grants SFRH/BPD/70574/2010 and SFRH/BPD/66958/2009 from FCT (Portugal), respectively. V.Zh.A. would further like to thank for the support by the ERC under the FP7/EC through a Starting Grant agreement number 239953. This work was made possible in part by a research grant from the Armenian National Science and Education Fund (ANSEF) based in New York, USA. Special thanks to Richard Trilling, who kindly agreed to edit the manuscript. This research made use of the Asiago Supernova Catalogue (ASC), which is available at http://web.oapd.inaf.it/supern/ cat/, the Sternberg Astronomical Institute (SAI) Supernova Catalogue, available at http://www.sai.msu.su/sn/sncat/, website of the Central Bureau for Astronomical Telegrams (CBAT), available at http://www.cbat.eps. harvard.edu/lists/Supernovae.html, the HyperLeda database (http:// leda.univ-lyon1. fr/), and the NASA/IPAC Extragalactic Database (NED), which is available at http://ned.ipac.caltech.edu/, and operated by the Jet Propulsion Laboratory, California Institute of Technology, under contract with the National Aeronautics and Space Administration. GAIA was created by the now closed Starlink UK project, funded by the Particle Physics and Astronomy Research Council (PPARC) and has been more recently supported by the Joint Astronomy Center Hawaii funded again by PPARC and more recently by its successor organization the Science and Technology Facilities Council (STFC). The GAIA home page is http://astro.dur. ac.uk/ pdraper/gaia/gaia.html. Funding for SDSS-III has been provided by the Alfred P. Sloan Foundation, the Participating Institutions, the National Science Foundation, and the US Department of Energy Office of Science. The SDSS-III web site is http://www.sdss3.org/. SDSS-III is managed by the Astrophysical Research Consortium for the Participating Institutions of the SDSS-III Collaboration including the University of Arizona, the Brazilian Participation Group, Brookhaven National Laboratory, University of Cambridge, University of Florida, the French Participation Group, the German Participation Group, the Instituto de Astrofisica de Canarias, the Michigan State/Notre Dame/JINA Participation Group, Johns Hopkins University, Lawrence Berkeley National Laboratory, Max Planck Institute for Astrophysics, New Mexico State University, New York University, Ohio State University, Pennsylvania State University, University of Portsmouth, Princeton University, the Spanish Participation Group, University of Tokyo, University of Utah, Vanderbilt University, University of Virginia, University of Washington, and Yale University.

\section{References}

Aihara, H., Allende Prieto, C., An, D., et al. 2011, ApJS, 193, 29 Aldering, G., Lee, B. C., Loken, S., et al. 2005, The Astronomer's Telegram, 451,1

Alongi, M., Bertelli, G., Bressan, A., et al. 1993, A\&AS, 97, 851

Anderson, J. P., \& James, P. A. 2008, MNRAS, 390, 1527

Anderson, J. P., \& James, P. A. 2009, MNRAS, 399, 559

Arcavi, I., Gal-Yam, A., Kasliwal, M. M., et al. 2010, ApJ, 721, 777

Baillard, A., Bertin, E., de Lapparent, V., et al. 2011, A\&A, 532, A74

Baldwin, J. A., Phillips, M. M., \& Terlevich, R. 1981, PASP, 93, 5 (BPT)
Barbon, R., Buondí, V., Cappellaro, E., \& Turatto, M. 1999, A\&AS, 139, 531 Bartunov, O. S., Makarova, I. N., \& Tsvetkov, D. I. 1992, A\&A, 264, 428 Bartunov, O. S., Tsvetkov, D. Y., \& Filimonova, I. V. 1994, PASP, 106, 1276 Bassett, B., Becker, A., Bizyaev, D., et al. 2007a, Central Bureau Electronic Telegrams, 1098, 1

Bassett, B., Becker, A., Bizyaev, D., et al. 2007b, Central Bureau Electronic Telegrams, 1102, 1

Bettoni, D., Hjorth, J., \& Benetti, S. 1998, IAU Circ., 6911, 1

Blanton, M. R., Hogg, D. W., Bahcall, N. A., et al. 2003, ApJ, 592, 819

Boissier, S., \& Prantzos, N. 2009, A\&A, 503, 137

Bottinelli, L., Gouguenheim, L., Paturel, G., \& Teerikorpi, P. 1995, A\&A, 296, 64

Bressan, A., Fagotto, F., Bertelli, G., \& Chiosi, C. 1993, A\&AS, 100, 647

Bressan, A., Della Valle, M., \& Marziani, P. 2002, MNRAS, 331, L25

Bruzual, G., \& Charlot, S. 2003, MNRAS, 344, 1000

Cappellaro, E., \& Turatto, M. 1988, A\&A, 190, 10

Cappellaro, E., Turatto, M., Tsvetkov, D. Y., et al. 1997, A\&A, 322, 431

Cappellaro, E., Evans, R., \& Turatto, M. 1999, A\&A, 351, 459

Cardelli, J. A., Clayton, G. C., \& Mathis, J. S. 1989, ApJ, 345, 245

Chabrier, G. 2003, PASP, 115, 763

Cherepashchuk, A. M., Metlova, N., Wheeler, J. C., et al. 1987, IAU Circ., 4381, 1

Cid Fernandes, R., González Delgado, R. M., Schmitt, H., et al. 2004, ApJ, 605, 105

Cid Fernandes, R., Mateus, A., Sodré, L., Stasińska, G., \& Gomes, J. M. 2005, MNRAS, 358, 363

Cid Fernandes, R., Stasińska, G., Schlickmann, M. S., et al. 2010, MNRAS, 403, 1036

Cid Fernandes, R., Stasińska, G., Mateus, A., \& Vale Asari, N. 2011, MNRAS, 413,1687

Condon, J. J., Cotton, W. D., Greisen, E. W., et al. 1998, AJ, 115, 1693

de Vaucouleurs, G., de Vaucouleurs, A., Corwin, Jr., H. G., et al. 1991, Third Reference Catalogue of Bright Galaxies, Vol. I: Explanations and references, Vol. II: Data for galaxies between $0^{\mathrm{h}}$ and $12^{\mathrm{h}}$, Vol. III: Data for galaxies between $12^{\mathrm{h}}$ and $24^{\mathrm{h}}$

Fagotto, F., Bressan, A., Bertelli, G., \& Chiosi, C. 1994a, A\&AS, 104, 365

Fagotto, F., Bressan, A., Bertelli, G., \& Chiosi, C. 1994b, A\&AS, 105, 29

Filippenko, A. V., \& Chornock, R. 2000, IAU Circ., 7511, 2

Filippenko, A. V., \& Chornock, R. 2002, IAU Circ., 7825, 1

Filippenko, A. V., \& Matheson, T. 1993, IAU Circ., 5842, 2

Filippenko, A. V., Li, W. D., \& Modjaz, M. 1999, IAU Circ., 7152, 2

Filippenko, A. V., Silverman, J. M., Foley, R. J., et al. 2007, Central Bureau Electronic Telegrams, 1101, 1

Förster, F., \& Schawinski, K. 2008, MNRAS, 388, L74

Frieman, J. A., Bassett, B., Becker, A., et al. 2008, AJ, 135, 338

Gelman, A., \& Rubin, D. B. 1992, Statistical Science, 7, 457

Girardi, L., Bressan, A., Chiosi, C., Bertelli, G., \& Nasi, E. 1996, A\&AS, 117, 113

Gunn, J. E., Siegmund, W. A., Mannery, E. J., et al. 2006, AJ, 131, 2332

Gyulzadyan, M., McLean, B., Adibekyan, V. Z., et al. 2011, Astrophys., 54, 15

Hakobyan, A. A. 2008, Astrophys., 51, 69

Hakobyan, A. A., Petrosian, A. R., McLean, B., et al. 2008, A\&A, 488, 523

Hakobyan, A. A., Mamon, G. A., Petrosian, A. R., Kunth, D., \& Turatto, M. 2009, A\&A, 508, 1259

Hakobyan, A. A., Petrosian, A. R., Mamon, G. A., et al. 2011, Astrophys., 54, 301

Han, D.-H., Park, C., Choi, Y.-Y., \& Park, M.-G. 2010, ApJ, 724, 502

Herrero-Illana, R., Pérez-Torres, M. Á., \& Alberdi, A. 2012, A\&A, 540, L5

Hubble, E. P. 1926, ApJ, 64, 321

Humphreys, R. M., Prieto, J. L., Rosenfield, P., et al. 2010, ApJ, 718, L43

Hutchings, D., \& Li, W. D. 2001, IAU Circ., 7724, 1

Iye, M., \& Kodaira, K. 1975, PASJ, 27, 411

Jester, S., Schneider, D. P., Richards, G. T., et al. 2005, AJ, 130, 873

Kasliwal, M. M., Kulkarni, S. R., Quimby, R., et al. 2009, Central Bureau Electronic Telegrams, 1819, 1

Kauffmann, G., Heckman, T. M., Tremonti, C., et al. 2003, MNRAS, 346, 1055 (K03)

Kaviraj, S., Schawinski, K., Devriendt, J. E. G., et al. 2007, ApJS, 173, 619

Kaviraj, S., Khochfar, S., Schawinski, K., et al. 2008, MNRAS, 388, 67

Kaviraj, S., Peirani, S., Khochfar, S., Silk, J., \& Kay, S. 2009, MNRAS, 394, 1713

Kawabata, K. S., Maeda, K., Nomoto, K., et al. 2010, Nature, 465, 326

Kazarian, M. A. 1997, Astrophys., 40, 296

Kelly, P. L., \& Kirshner, R. P. 2011, ApJ, submitted [arXiv: 1110. 1377]

Kewley, L. J., Dopita, M. A., Sutherland, R. S., Heisler, C. A., \& Trevena, J. 2001, ApJ, 556, 121 (K01)

Kewley, L. J., Jansen, R. A., \& Geller, M. J. 2005, PASP, 117, 227 
A. A. Hakobyan et al.: Supernovae and their host galaxies. I.

Kewley, L. J., Groves, B., Kauffmann, G., \& Heckman, T. 2006, MNRAS, 372, 961

Kushida, R., Nakano, S., Kushida, Y., \& Aoki, M. 1998, IAU Circ., 6920, 2

Leaman, J., Li, W., Chornock, R., \& Filippenko, A. V. 2011, MNRAS, 412, 1419

Leloudas, G., Gallazzi, A., Sollerman, J., et al. 2011, A\&A, 530, A95

Leonard, D. C. 2010, The Astronomer's Telegram, 2750, 1

Lennarz, D., Altmann, D., \& Wiebusch, C. 2012, A\&A, 538, A120

Li, W., Wang, X.-F., \& Bian, F.-Y. 2005, Central Bureau Electronic Telegrams, 332,1

Li, W., Chornock, R., Leaman, J., et al. 2011, MNRAS, 412, 1473

Maksym, A., Elenin, L., \& Schwartz, M. 2010, Central Bureau Electronic Telegrams, 2245, 1

Mannucci, F., Della Valle, M., Panagia, N., et al. 2005, A\&A, 433, 807

Matheson, T., Jha, S., Challis, P., Kirshner, R., \& Calkins, M. 2001, IAU Circ., 7597, 3

Maund, J. R., Smartt, S. J., Kudritzki, R.-P., et al. 2006, MNRAS, 369, 390

Maxwell, A. J., Graham, M. L., Parker, A., et al. 2010, Central Bureau Electronic Telegrams, 2245, 2

Miknaitis, G., Pignata, G., Rest, A., et al. 2007, ApJ, 666, 674

Modjaz, M., Kirshner, R., Challis, P., Blondin, S., \& Berlind, P. 2005, IAU Circ., 8650,2

Modjaz, M., Kewley, L., Bloom, J. S., et al. 2011, ApJ, 731, L4

Naim, A., Lahav, O., Buta, R. J., et al. 1995, MNRAS, 274, 1107

Nair, P. B., \& Abraham, R. G. 2010, ApJS, 186, 427

Navasardyan, H., Petrosian, A. R., Turatto, M., Cappellaro, E., \& Boulesteix, J. 2001, MNRAS, 328, 1181

Nugent, P., Aldering, G., Castro, P., Nunes, N., \& Quimby, R. 1998, IAU Circ., 6804, 1

Pastorello, A. 2012, Mem. Soc. Astron. Ital. Suppl., 19, 24

Patat, F., Barbon, R., Cappellaro, E., \& Turatto, M. 1997, A\&A, 317, 423

Patat, F., Pastorello, A., \& Aceituno, J. 2003, IAU Circ., 8167, 3

Paturel, G., Andernach, H., Bottinelli, L., et al. 1997, A\&AS, 124, 109

Paturel, G., Petit, C., Prugniel, P., et al. 2003, A\&A, 412, 45

Perets, H. B., Gal-Yam, A., Mazzali, P. A., et al. 2010, Nature, 465, 322

Perets, H. B., Gal-Yam, A., Crockett, R. M., et al. 2011, ApJ, 728, L36

Petrosian, V. 1976, ApJ, 209, L1

Petrosian, A. R., \& Turatto, M. 1990, A\&A, 239, 63

Petrosian, A. R., \& Turatto, M. 1995, A\&A, 297, 49

Petrosian, A., Navasardyan, H., Cappellaro, E., et al. 2005, AJ, 129, 1369

Petrosian, A., McLean, B., Allen, R. J., \& MacKenty, J. W. 2007, ApJS, 170, 33
Pojmanski, G. 2007, IAU Circ., 8875, 1

Prantzos, N., \& Boissier, S. 2003, A\&A, 406, 259

Prieto, J. L., Stanek, K. Z., \& Beacom, J. F. 2008, ApJ, 673, 999

Puckett, T., Peoples, M., Joubert, N., et al. 2006, IAU Circ., 8741, 1

Quimby, R., Hoeflich, P., Wheeler, J. C., et al. 2005, IAU Circ., 8504, 3

Riess, A. G., Macri, L., Casertano, S., et al. 2009, ApJ, 699, 539

Schaefer, B. E. 2001, IAU Circ., 7608, 1

Schlegel, D. J., Finkbeiner, D. P., \& Davis, M. 1998, ApJ, 500, 525

Smartt, S. J. 2009, ARA\&A, 47, 63

Smith, N., Miller, A., Li, W., et al. 2010, AJ, 139, 1451

Smith, N., Li, W., Silverman, J. M., Ganeshalingam, M., \& Filippenko, A. V. 2011, MNRAS, 415, 773

Spergel, D. N., Bean, R., Doré, O., et al. 2007, ApJS, 170, 377

Strauss, M. A., Weinberg, D. H., Lupton, R. H., et al. 2002, AJ, 124, 1810

Suh, H., Yoon, S.-C., Jeong, H., \& Yi, S. K. 2011, ApJ, 730, 110

Terry, J. N., Paturel, G., \& Ekholm, T. 2002, A\&A, 393, 57

Theureau, G., Rauzy, S., Bottinelli, L., \& Gouguenheim, L. 1998, A\&A, 340, 21

Tsvetkov, D. Y., Blinnikov, S. I., \& Pavlyuk, N. N. 2001, Astron. Lett., 27, 411

Tsvetkov, D. Y., Pavlyuk, N. N., \& Bartunov, O. S. 2004, Astron. Lett., 30, 729

Turatto, M. 2003, in Supernovae and Gamma-Ray Bursters, ed. K. Weiler, Lecture Notes in Physics (Berlin: Springer Verlag), 598, 21

Turatto, M., Benetti, S., \& Pastorello, A. 2007, in Supernova 1987A: 20 Years After: Supernovae and Gamma-Ray Bursters, eds. S. Immler, K. Weiler, \& R. McCray, AIP Conf. Ser., 937, 187

Uomoto, A., Smee, S., Rockosi, C., et al. 1999, in BAAS 31, AAS Meeting Abstracts, 1501

van den Bergh, S. 1988, PASP, 100, 344

van den Bergh, S. 1997, AJ, 113, 197

van den Bergh, S., \& Tammann, G. A. 1991, ARA\&A, 29, 363

van den Bergh, S., Li, W., \& Filippenko, A. V. 2002, PASP, 114, 820

van den Bergh, S., Li, W., \& Filippenko, A. V. 2003, PASP, 115, 1280 van den Bergh, S., Li, W., \& Filippenko, A. V. 2005, PASP, 117, 773

van Dyk, S. D., Hamuy, M., \& Filippenko, A. V. 1996, AJ, 111, 2017 van Dyk, S. D., Peng, C. Y., King, J. Y., et al. 2000, PASP, 112, 1532

Wagner, R. M., Vrba, F. J., Henden, A. A., et al. 2004, PASP, 116, 326

Wang, J., \& Wei, J. Y. 2008, ApJ, 679, 86

Wang, J., Deng, J. S., \& Wei, J. Y. 2010, MNRAS, 405, 2529

Wild, P., \& Schildknecht, T. 1987, IAU Circ., 4374, 1

Yahil, A., Tammann, G. A., \& Sandage, A. 1977, ApJ, 217, 903 